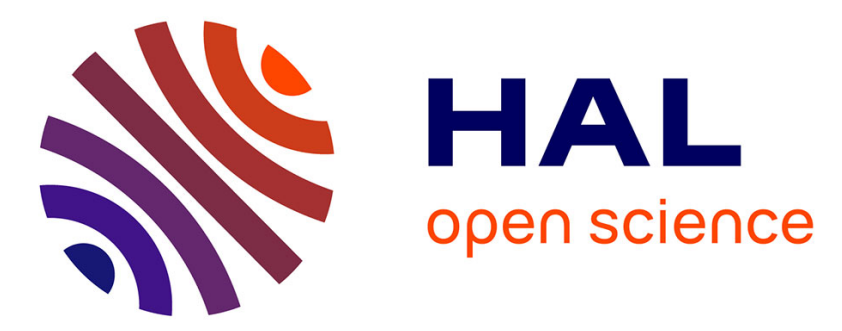

\title{
Positive plant-soil feedback trigger tannin evolution by niche construction: A spatial stoichiometric model
}

\author{
Jean-François Arnoldi, Sylvain Coq, Sonia Kéfi, Sébastien Ibanez
}

\section{To cite this version:}

Jean-François Arnoldi, Sylvain Coq, Sonia Kéfi, Sébastien Ibanez. Positive plant-soil feedback trigger tannin evolution by niche construction: A spatial stoichiometric model. Journal of Ecology, 2019, 10.1111/1365-2745.13234 . hal-02331461

\section{HAL Id: hal-02331461 \\ https://hal.umontpellier.fr/hal-02331461}

Submitted on 16 Nov 2020

HAL is a multi-disciplinary open access archive for the deposit and dissemination of scientific research documents, whether they are published or not. The documents may come from teaching and research institutions in France or abroad, or from public or private research centers.
L'archive ouverte pluridisciplinaire HAL, est destinée au dépôt et à la diffusion de documents scientifiques de niveau recherche, publiés ou non, émanant des établissements d'enseignement et de recherche français ou étrangers, des laboratoires publics ou privés. 


\title{
Positive plant-soil feedbacks trigger tannin evolution by niche construction: a spatial stoichiometric model
}

\author{
Jean-François Arnoldi ${ }^{1}$, Sylvain $\mathrm{Coq}^{2}$, Sonia Kéfi ${ }^{3}$, and Sébastien Ibanez ${ }^{4,}$
}

1. Zoology department, School of Natural Sciences, Trinity College Dublin, Ireland.

2. Centre d'Écologie Fonctionnelle et Évolutive (CEFE) UMR 5175, Université de Montpellier - CNRS - Université Paul-Valéry Montpellier - EPHE, Montpellier, France.

3. Institut des Sciences de l'Évolution de Montpellier, CNRS, Université de Montpellier, IRD, EPHE, CC 065, Montpellier, France.

4. Laboratoire d'Écologie Alpine (LECA), CNRS UMR 5553, Université Savoie Mont Blanc, Chambéry, France.

* Corresponding author

Keywords: coevolution, eco-evolutionary dynamics, mycorrhizae, nutrient cycling, plantherbivore interaction, plant secondary metabolites, spatial dynamics.

Manuscript type: Research Article. 


\section{Abstract}

1. Among plant traits, plant secondary metabolites such as tannins mediate plantherbivore interactions but also have after-life effects on litter decomposition and nutrient cycling. We propose that niche construction mechanisms based on positive plant-soil feedbacks (PSF) could influence the evolution of tannin production.

2. By modeling the flow of nitrogen and carbon through plants and soil in a spatially explicit context, we explored the relative contribution of herbivory and positive PSF as drivers of tannin evolution. We assumed soil nitrogen to be composed of labile and recalcitrant compartments, the latter made of tannin-protein complexes accessible by plants via associations with mycorrhizal fungi.

3. In infertile environments and for plants with low biomass turnover rates, we show that when tannins modify soil properties locally, positive PSF alone can drive their evolution. We further predict the existence of positive coevolutionary feedbacks between associations with mycorrhizae and tannins, possibly triggered by the evolution of the latter as protection against herbivores. In line with our theoretical results, empirical evidence suggest that tannins are mostly present in plants with low tissue turnover, associated with mycorrhizae able to decay organic matter and inhabiting infertile environments.

4. Synthesis. Our model proposes that the evolution of tannin production can be triggered by positive PSF, provided that tannins locally increase the amount of recalcitrant nitrogen and that mycorrhizal fungi associated with plants are able to reabsorb nitrogen from tannin-protein complexes. In our model, tannin production evolves only in infertile ecosystems, in agreement with field observations. Our findings highlight that the strength of niche construction depends on the ecological context, i.e. that global ecological properties constrain local eco-evolutionary dynamics. 


\section{Introduction}

Organisms change (or construct) their environment and those changes in turn modify the selective pressures to which organisms are exposed. The modified selection pressures then trigger the evolution of traits which may cause further change in the environment, and so on. This process, in which organisms and environment are both subjects and objects (Lewontin, 1983), is called niche construction (Odling-Smee et al., 2003). Many plant traits may have evolved because of their niche construction abilities, including flammability (Schwilk, 2003), germination timing (Donohue et al., 2005) and plant-soil feedbacks (PSF) (Schweitzer et al., 2014). Some PSF are negative, e.g. plants can locally increase pathogen density (Putten et al., 2013), leading to a Janzen-Connell effect. On the other hand, positive PSF also occur (Klironomos, 2002; Putten et al., 2013; Van Nuland et al., 2019) as plants may enhance the density of their mutualists (Liang et al., 2015) or because the decomposition of their litter is faster in their own soil (Ayres et al., 2009). In either case, many components of a plant's environment are determined by the plant itself, each plant genotype shaping its own environment (Schweitzer et al., 2004; Whitham et al., 2006). In turn, these altered environmental conditions will affect the selective pressures acting on plant traits, thereby constituting an ecological-evolutionary feedback loop (Van Nuland et al., 2016), i.e. a reciprocal interaction between ecological and evolutionary processes (Govaert et al., 2019).

However, organisms construct their environment only to a certain extent, and many environmental factors are not (or hardly) modified by their actions. Such environmental factors may either prevent or enhance the evolution of niche-constructing traits. For instance, flammability will more likely evolve in dry rather than wet environments. Similarly, the magnitude and direction of ecological-evolutionnary loops based on PSF are expected to vary along soil gradients (Van Nuland et al., 2016). Most models of niche construction, however, do not include the interplay between environmental factors and niche constructing traits (Kylafis and Loreau, 2008; Kéfi et al., 2008; Laland et al., 1999; 
Lehmann, 2008; Schweitzer et al., 2014). Here, we propose that the evolution of tannin production in plants is a useful case-study to focus on this interplay. We argue that tannins may trigger positive PSF because they complex organic nitrogen and limit nutrient losses, thereby creating a locally enriched soil. Furthermore, we assert that the evolutionary importance of such feedbacks depends on the fertility of the environment.

Among the large variety of plant's defensive structures and compounds, tannins are a widely distributed class of plant secondary metabolites (Mole, 1993), with a wide array of intra- and interspecific variation in their production (Kraus et al., 2003). Tannins have been defined as water-soluble phenolic compounds, with a molecular mass between 0.5 and $3 \mathrm{kDa}$ and able to precipitate proteins and alkaloids (Bate-Smith and Swain, 1962). Two major types of tannins, condensed tannins and hydrolysable tannins, have been distinguished based on their respective chemistry, yet both types share the ability to complex proteins. Although tannins have been extensively studied as anti-herbivore defense (Bergvall and Leimar, 2005; Bernays et al., 1989; Feeny, 1970; Hagerman and Butler, 1991), they also affect nutrient cycling. Tannins enter the soil as a component of litter, impacting nutrient dynamics (Hättenschwiler and Vitousek, 2000; Joanisse et al., 2009; Kraus et al., 2003; Northup et al., 1995), limiting leaching (Jordan et al., 1979; Northup et al., 1995) and slowing-down decomposition and microbial activities (Benoit and Starkey, 1968; Coq et al., 2010; Joanisse et al., 2007; Schweitzer et al., 2004). Emphasis on the evolutionary role of either properties (defence or nutrient cycling) has been referred to as green foliage and litter perspectives, respectively (Hättenschwiler et al., 2011).

From the green foliage perspective, tannins production evolves as an anti-herbivore strategy, because tannins decrease plants palatability. Once in the soil, the complexation of proteins by tannins triggers the formation of recalcitrant organic matter, from which nutrient accessibility is reduced. From the green foliage perspective, the reduction of resource availability due to the after-life effects of tannins is a detrimental side-effect of an anti-herbivore strategy. However, from the litter perspective the after-life effects of 
tannin production may instead benefit plants through positive PSF. Indeed, complexation of proteins enables local nutrient retention, which could be advantageous if plants can retrieve nutrients directly from recalcitrant organic matter, thereby circumventing mineralization by free microorganisms (Aerts and Chapin, 2000; Northup et al., 1995). Mycorrhizal associations with symbiotic fungi can grant this ability to plants. This is the case, in particular, for plants associated with ericoid mycorrhizae (Bending and Read, 1996; Joanisse et al., 2009; Wurzburger and Hendrick, 2009), which abound in heathland and peatlands. For such mycorrhizal associations, tannin production could thus be a major process by which plants control their own resources, retaining nutrients in the local environment of the plant.

This raises the question of the ecological conditions that could promote the role of positive PSF in the evolution of tannin production. The observed correlation between nutrient limitation and tannin production in plants (Endara and Coley, 2011; McKey et al., 1978) could be the result of a strategy to cope with an infertile environment by limiting nutrient losses through the formation of protein-tannin complexes. But this is not selfevident. In fact, based on an eco-evolutionary model, Barot et al. (2014) suggested that plants should evolve fast decomposing litter in nutrient-poor environments, in apparent contradiction with empirical findings (Ordoñez et al., 2009). Because tannins slow down mineralization, the question of whether the afterlife effects of tannins are beneficial or detrimental to plants in infertile ecosystems is thus still open. If beneficial, tannin evolution could be driven by positive PSF alone, in line with the litter perspective. Additional selective pressure due to the protection against herbivory would then be unnecessary, although compatible. If detrimental, tannin evolution would instead be the outcome of a trade-off between anti-herbivore defense and the reduction of nutrient cycling, in line with the green foliage perspective.

Here we address this question and reconcile observations and theoretical predictions. To do so, we developed and analyzed a mechanistic evolutionary model, which includes 
the effects of tannins on both herbivory and nutrient-based PSF. Because tannins are carbon (C) based compounds, and because their afterlife effect notably affects nitrogen (N) cycling, our model explicitly accounts for plants C:N stoichiometry. Because tanninprotein complexes are insoluble, immobile and remain near the tannin-producing plants, our model accounts for spatial structure. Plant associations with specific mycorrhizae will be able to reabsorb $\mathrm{N}$ from tannin-protein complexes, in addition to labile N. Our model thus describes the ecological dynamics of plants growing in an N-limited substrate, plus the evolutionary dynamics of tannin production and symbiotic associations with mycorrhizae, in a stoichiometric and spatial context.

To guide our investigation, we formulate three hypothesis. In the first two, it is assumed that all plants can absorb recalcitrant $\mathrm{N}$, with equal and constant symbiotic capacity, so that positive PSF can take place. We then propose that:

Hypothesis 1. Local positive PSF can trigger tannin evolution, even in the absence of herbivory. Hence, a well-mixed version of the model will impede tannin evolution in the absence of herbivory, while spatial structure will allow tannin evolution.

Hypothesis 2. Tannin evolution through positive PSF is more likely in infertile than in fertile ecosystems. This can be tested theoretically by varying a model parameter representing soil fertility.

The third and last hypothesis is that the existence of positive PSF via mychorrizal association - assumed in hypothesis 1 and 2 - can be justified from evolutionary principles. More specifically, we propose that:

Hypothesis 3. There exists a positive coevolutionary feedback between symbiotic association with mycorrhizal fungi and tannin production, which can be triggered by herbivores. Tannin production will not evolve under ecological conditions which preclude symbiotic association in the first place, unless tannin production initially evolves as a response to herbivory. In this case the evolution of symbiotic associations will follow, and further enhance the selective pressure towards tannin production. We test this hy- 
pothesis with a coevolutionary version of our model, where two phenotypic traits evolve, namely plant symbiotic capacity and plant tannin content.

\section{Materials and Methods}

In our model, $\mathrm{C}$ is acquired by plants through photosynthesis, and $\mathrm{N}$ from the soil, where it can be in either labile or recalcitrant form. Labile $\mathrm{N}$ is available from the environment but is also recycled through the decomposition of tannin-free plant litter. By contrast, recalcitrant $\mathrm{N}$ is composed of tannin-protein complexes and is only produced by tannin producing plants. We do not explicitly model a litter compartment. However, labile $\mathrm{N}$ implicitly comes from low $\mathrm{C}: \mathrm{N}$ and low tannin litter, while recalcitrant $\mathrm{N}$ would be found in high tannin, and thus high $\mathrm{C}: \mathrm{N}$, litter.

Labile $\mathrm{N}$ can be absorbed by all plants, whereas recalcitrant $\mathrm{N}$ can only be absorbed if plants are associated with mycorrhizae, which comes at a cost in C. Hereafter, the specific mycorrhizae that have the ability to access $\mathrm{N}$ from complex nitrogen sources such as tannin-protein complexes will simply be referred to as "mycorrhizae", and "symbiotic capacity" will refer to the investment of plants into these fungal partners.

Finally, herbivores remove plant biomass (and thus accelerate turnover). Because tannins deter herbivores, herbivory is weaker when plants produce tannins. Two plant traits are evolving in our model: the proportion of tannins in plant biomass $(\tau)$, and the symbiotic capacity (e) to associate with mycorrhizae. We implemented the model at two levels of spatial complexity: a mean-field model assuming plants and soil to be well mixed in the landscape, and a spatially explicit model using a cellular automaton, with stochastic birth and death events.

Below we explain the model focusing on ecological intuitions. Mathematical details and a table of parameters can be found in Appendix A and B. 
A.

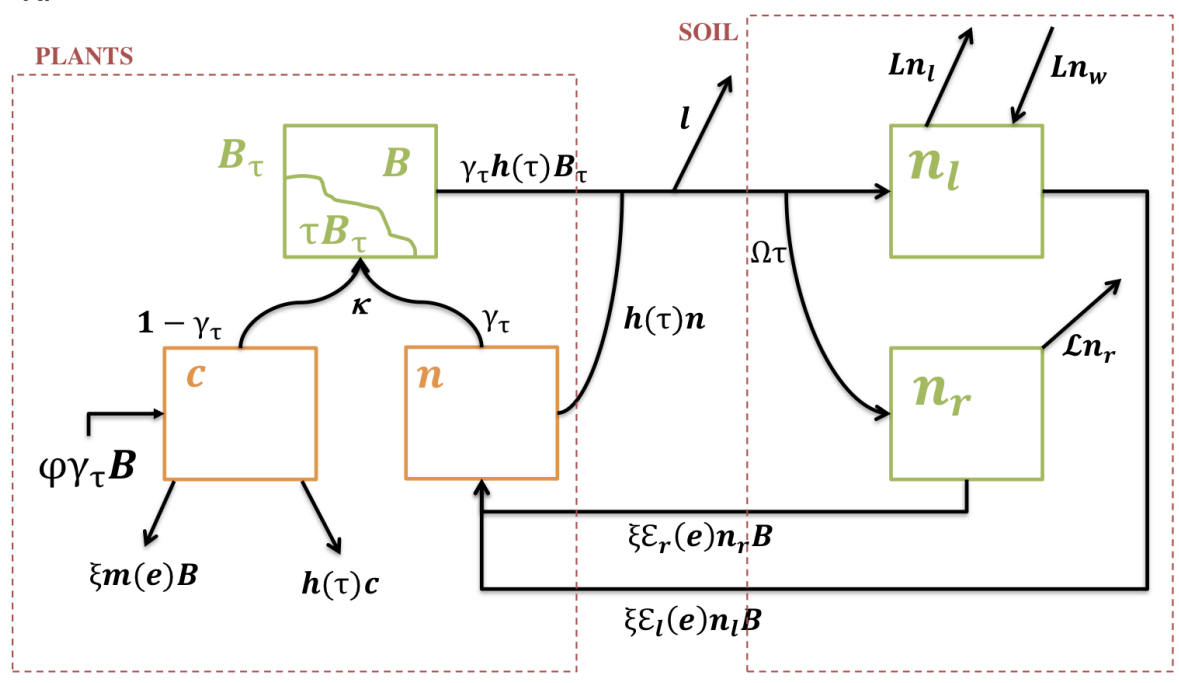

B.

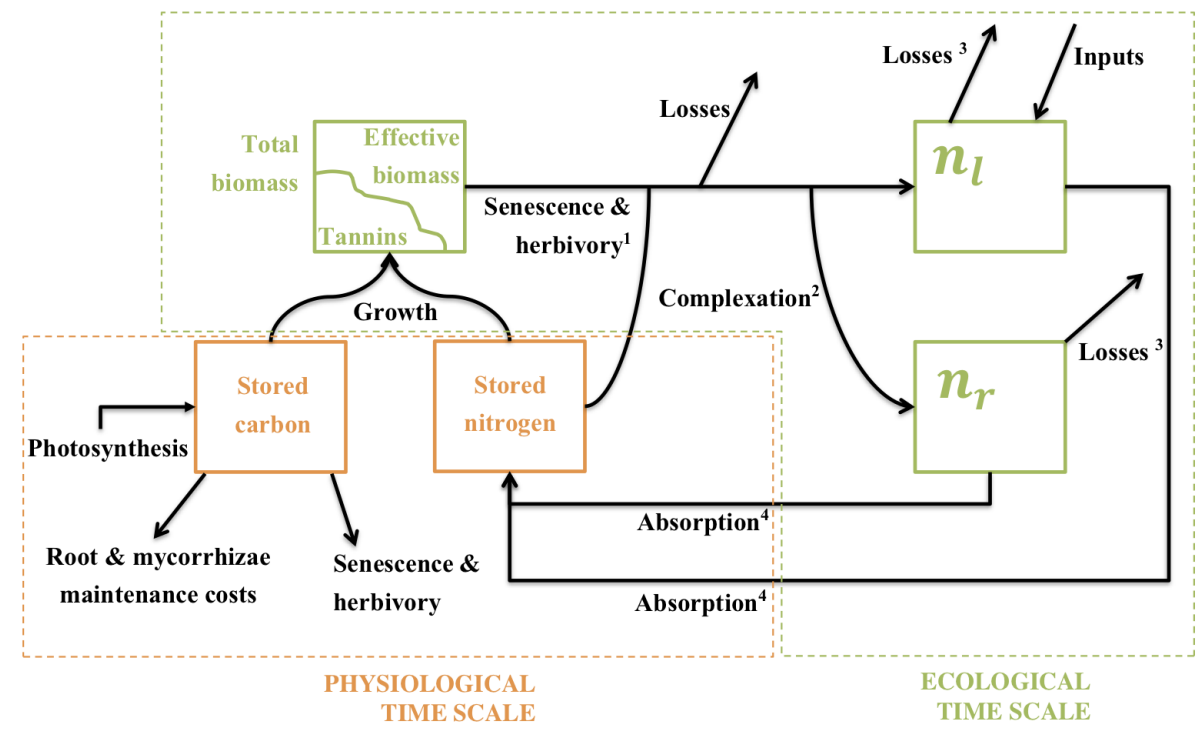

Figure 1: Scheme of the model for plant growth. (A) symbols, (B) words and references. (A) Left: the three plant compartments, Right: the two soil compartments. (B) Carbon $(c)$ and nutrient $(n)$ stocks are used for the growth of plant biomass $\left(B_{\tau}\right)$, which includes tannins. Following senescence, nutrients enter one of the two soil compartments. Nutrients complexed by tannins enter the recalcitrant nutrient pool $\left(n_{r}\right)$, the remaining nutrients enter the labile nutrient pool $\left(n_{l}\right)$. Nutrients are then absorbed by plants, at the expense of carbon costs. ${ }^{1}$ Bernays et al. (1989); Feeny (1970); Hagerman and Butler (1991); ${ }^{2}$ Hättenschwiler and Vitousek (2000); Northup et al. (1995); ${ }^{3}$ Northup et al. (1998); ${ }^{4}$ Joanisse et al. (2009); Wu et al. (2003). 


\section{Plant growth}

We denote $B_{\tau}$ the $\mathrm{C}+\mathrm{N}$ content of plant biomass with a C:N ratio of $\alpha_{\tau}$ (not counting $\mathrm{C}$ and $\mathrm{N}$ stocks that are used to create $B_{\tau}$, see below). A fraction $\tau$ is used to produce tannins, whereas the rest, $B$, is involved in all other physiological processes, like photosynthesis, nutrient absorption or $C$ exchange with mycorrhizae (we thus call $B$ the "effective" biomass). We denote $\gamma_{\tau}$ the proportion of $\mathrm{N}$ in $B_{\tau}$, with $1-\gamma_{\tau}$, representing the complementary proportion of $\mathrm{C}$. Biomass growth is controlled by the limiting factor among $\mathrm{C}$ and $\mathrm{N}$ stocks in plant cells, denoted $n$ and $c$. Mathematically, biomass growth is therefore controlled by the minimum between $\frac{n}{\gamma_{\tau}}$ and $\frac{c}{1-\gamma_{\tau}}$ (Danger et al., 2008; Odum, 1971). We further assume a constant plant tissue turnover rate $h$, so that plant biomass $B_{\tau}$ follows

$$
\frac{1}{\mu} \frac{d B_{\tau}}{d t}=\underbrace{\min \left\{\frac{n}{\gamma_{\tau}}, \frac{c}{1-\gamma_{\tau}}\right\}}_{\text {growth }}-\underbrace{h B_{\tau}}_{\text {turnover and herbivory }}
$$

Here $\mu$ is a rate, which sets plants' ecological timescale. Herbivory decreases plant biomass, as a part of the global turnover rate $h$, so that $h$ is a decreasing function of $\tau$ when herbivores are present.

\section{Resource acquisition}

In the soil, $\mathrm{N}$ is divided in two compartments: labile $n_{l}$, and recalcitrant $n_{r}$. Recalcitrant $\mathrm{N}$ is produced by plants themselves when tannins complex $\mathrm{N}$-containing proteins during leaf senescence (Northup et al., 1995). A critical assumption of our model is that symbiotic associations with mycorrhizae are necessary to absorb recalcitrant $\mathrm{N}$ (Pritsch and Garbaye, 2011; Read and Perez-Moreno, 2003; Wurzburger and Hendrick, 2009). We modeled this requirement by defining labile and recalcitrant absorption efficiencies, $\mathcal{E}_{l}$ and $\mathcal{E}_{r}$ as functions of symbiotic capacity $e$. We construct these functions so that symbiotic capacity enhances the absorption of both labile and recalcitrant nitrogen, and is necessary to absorb recalcitrant $\mathrm{N}$ (see Appendix A). Finally, we assumed that being 
associated with mycorrhizae comes at a carbon cost of maintenance $m$.

The dynamics of plant stocks ( $n$ and $c$ ) are the product of nutrient absorption efforts and carbon costs, with carbon absorbed by photosynthesis, at a rate $\varphi$. We assume that these physiological dynamics are faster than ecological dynamics, and that plants constantly adapt their absorption effort to remain at stoichiometric balance. We show in Appendix A that, under these assumptions, Eq. (1) becomes:

$$
\frac{1}{\mu} \frac{d B_{\tau}}{d t}=(P-h) B_{\tau}
$$

Where productivity $P$ is proportional to nutrient absorption, following a type-II functional response to soil $\mathrm{N}$ content $\left(n_{l}\right.$ and $n_{r}$, the latter a function of tannin content):

$$
P \approx \sum_{i=l, r} \mathcal{A}_{i}=\varphi \sum_{i=l, r} \frac{\frac{\mathcal{E}_{i} n_{i}}{m}}{1+\alpha_{\tau} \sum_{i} \frac{\mathcal{E}_{i} n_{i}}{m}}
$$

Here the $\mathcal{A}_{l}$ and $\mathcal{A}_{r}$ represent the absorption rates of labile and recalcitrant $\mathrm{N}$, respectively. They are functions of photosynthesis $\varphi$, soil $\mathrm{N}$ content, $n_{i}$, the ratios of absorption efficiencies over costs $\mathcal{E}_{i} / m$ (and thus symbiotic capacity $e$ ), and depend, finally, on the plant C:N ratio $\alpha_{\tau}$, which is larger if plants produce tannins.

\section{Nutrient absorption and recycling}

The dynamics of labile nutrients $n_{l}$ are controlled by three fluxes: absorption by plants, input by litter fall, and exogenous nutrient flows. Combined, this leads to

$$
\frac{d n_{l}}{d t}=\mu(\underbrace{\mathcal{R}_{l}}_{\text {recycling }}-\underbrace{\mathcal{A}_{l}}_{\text {absorption }}) \gamma B+\underbrace{L\left(n_{w}-n_{l}\right)}_{\text {in and out flows }}
$$

Absorption by plants removes $\mathrm{N}$ from the soil at a rate proportional to $\mathcal{A}_{l}$. Litter input depends (i) on the amount of litter entering the soil and (ii) on the proportion of litter $\mathrm{N}$ input that is in a labile form. The amount of litter $\mathrm{N}$ entering the soil is proportional to turnover rate $h$ and the proportion of $\mathrm{N}$ contained in plant biomass. (see Appendix $\mathrm{A}$ for an explicit expression). The proportion in labile form is a negative function of tannin 
content, because tannins will complex recycled $\mathrm{N}$, turning it into its recalcitrant form. The dynamics of labile soil nitrogen are completed by an exogenous flow, representing natural input and output of nitrogen with $n_{w}$ the bare soil nutrient content (Droop, 1968; Lemesle and Mailleret, 2008). Complexed $\mathrm{N}$ flows into the recalcitrant compartment $n_{r}$, whose dynamics read

$$
\frac{d n_{r}}{d t}=+\mu(\underbrace{\mathcal{\mathcal { R } _ { r }}}_{\text {recycling }}-\underbrace{\mathcal{\mathcal { A } _ { r }}}_{\text {absorption }}) \gamma B-\underbrace{\mathcal{L} n_{r}}_{\text {leaching }}
$$

Here the recalcitrant leaching rate $\mathcal{L}$, because of complexation by tannins, is smaller than the one of labile nutrients $L$. In the mean-field plant-soil model, space plays no role (plants are well mixed in the landscape) so that biomass growth at the population level, and soil dynamics at the landscape-level, are simply defined by Eqs. (2-4-5).

\section{Patch dynamics}

Because PSF act at a local scale, we accounted for spatial effects using a cellular automaton. For simplicity, space was represented as a linear landscape of $\mathrm{S}$ patches, which can be either empty or occupied by an individual plant (see Appendix B). However, our general conclusions will hold for more general spatial structures. This is because the niche-construction mechanisms that we investigate only rely on a notion of locality (Zelnik et al., 2019), thereby enabling individuals to benefit form the transformation of their local resource environment. Individual plants grow according to Eqs. (2-4-5), absorbing and recycling $\mathrm{N}$ locally. But, in contrast to the mean-field model, soil $\mathrm{N}$ diffuses continuously across patches. We assume labile $\mathrm{N}$ diffusion to be much larger than recalcitrant $\mathrm{N}$ diffusion, making recalcitrant $\mathrm{N}$ less mobile. Population dynamics is determined by a recruitment-death process. We assume that larger individuals are less likely to die than smaller ones and that the production of seedlings increases with biomass. We assumed the recruitment of a seedling in an empty patch to be higher near the parent plant than in more distant patches. Demographic parameters were tuned to always have a densely 


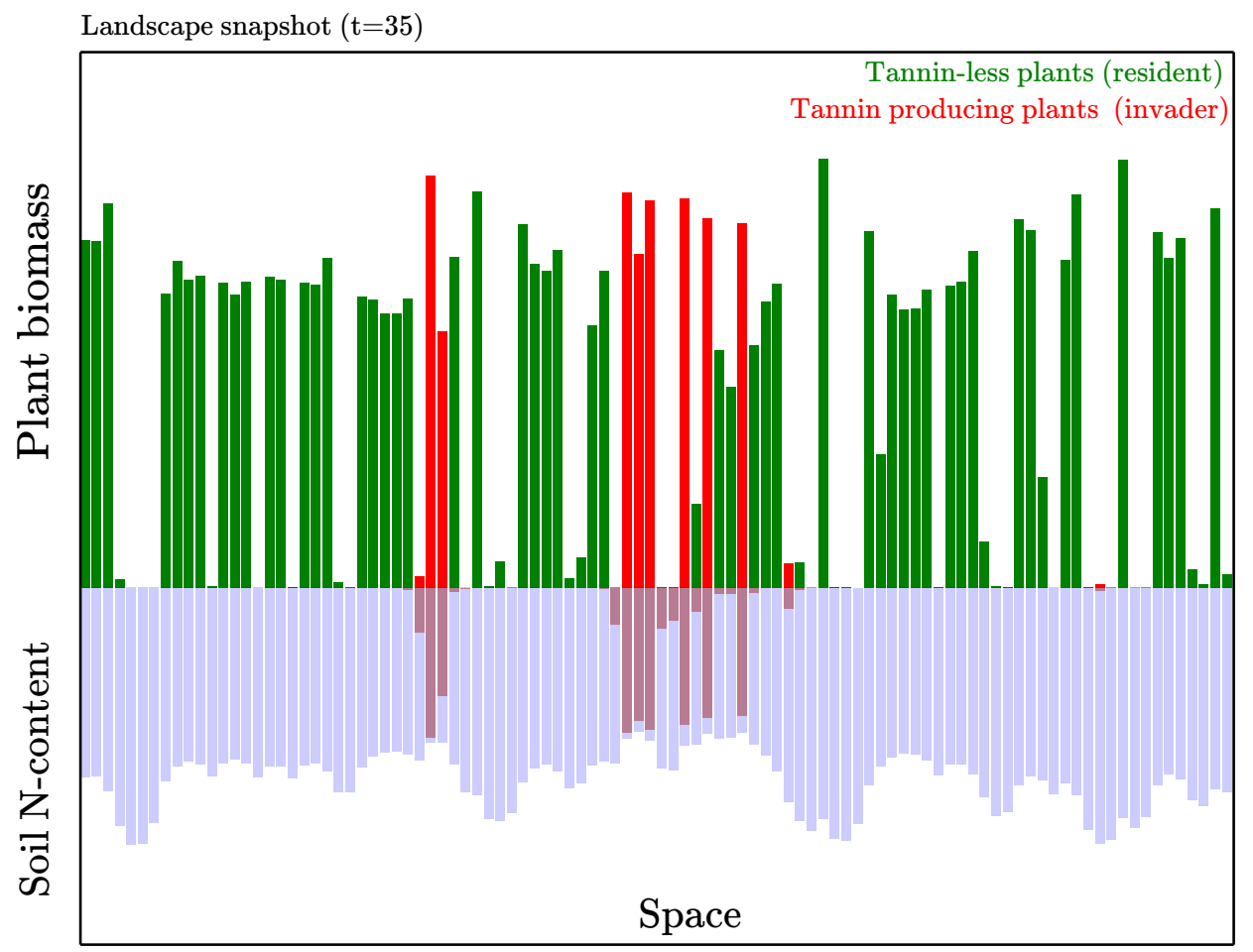

Figure 2: The cellular automaton (snapshot). Each green or red bar represent the biomass of an individual plant. Inverted bars represent soil $\mathrm{N}$-content in its labile (light purple) and recalcitrant form (dark red). We follow here the invasion attempt of tannin producing plants (in red) in a landscape populated by a tannin-less resident population (in green).

populated landscape. Indeed, if many patches are empty at any given time, the dynamics become neutral and we cannot expect it to exhibit selective pressures (see Table A1 for a complete list of model parameters).

\section{Model analyses}

We study the conditions for tannin producing plants to invade a resident population of tannin-less plants. We first suppose that all plants can absorb recalcitrant $\mathrm{N}$, with equal and constant symbiotic capacity. We focus on the influence of two key parameters, the effective bare soil $\mathrm{N}$ content and the plants' relative turnover rate $h$. The effective bare 
soil $\mathrm{N}$ content, $\mathcal{N}$, is a nondimensional combination of parameters:

$$
\mathcal{N}=\frac{\mathcal{E}_{l} n_{w}}{m}
$$

It is a proxy for soil fertility, as perceived by plants. More precisely, effective bare soil $\mathrm{N}$ content is the product of nutrient enrichment $\left(n_{w}\right)$ and the benefit-to-cost ratio of absorption efficiency over maintenance costs $\left(\mathcal{E}_{l} / m\right)$ that determines how the soil Ncontent translates in terms of fertility. Changing $\mathcal{N}$ allows us to test if the model predicts that infertile environments favor tannin production, as observed in nature. Plants relative turnover $h$ is a proxy for plant life strategy. High $h$ plants will enhance nutrient cycling while low $h$ plants will slow it down. To study the invasibility of the tannin phenotype, our reasoning relies on the analysis of the invasion fitness function (Geritz et al., 1997). The latter is the initial growth rate of a mutant population in the presence of a resident population at equilibrium. In some simulations we directly monitor the outcome of an invasion attempt, but the basic idea remains the same. A resident population sets the state of soil labile $\mathrm{N}$ and available light that a rare mutant population $B_{\tau}$ will experience. The mutant's population initial growth rate, or fitness, is thus defined as

$$
W=\left.\frac{1}{B_{\tau}} \frac{d B_{\tau}}{d t}\right|_{B_{\tau}=0}
$$

evaluated at the equilibrium value of the resident population. The fitness gradient 248 $\left.\partial_{\tau} W\right|_{\tau=0}$ determines the invasibility of mutants: if positive, a mutation leading to a small change in the phenotype $\tau$ can spread via the exponential growth of the population of mutants, so that the phenotype is consequently selected. In our model, the fitness gradient is proportional to the difference between the change of nutrient absorption rate and the change in turnover rate, caused by tannin production:

$$
\partial_{\tau} W \propto \partial_{\tau} \mathcal{A}-\partial_{\tau} h
$$

If the phenotypic change increases the absorption rate $\mathcal{A}$ and/or decreases the turnover rate $h$, mutants will invade. In our model, tannins can be selected as a protection against 
herbivores because they decrease turnover. In the absence of herbivores the selective advantage of tannins can only come through an increase of absorption. This implies that mutants, by producing tannins, have transformed the soil composition to their advantage, so that the C-cost of tannin production is worth the benefits of an increase of the recalcitrant nutrient pool. Because mutants are rare by definition, this cannot happen if they are well mixed in the landscape.

In a first step we assume that mutants, via some spatial mechanisms such as aggregation, do modify their local $\mathrm{N}$-environment. We call this approach spatially implicit, as it does not explicitly address the origins of the local modification of the resource environment by mutants, but assume this modification to take place. We define a parameter $z$, that determines the amount of recalcitrant $\mathrm{N}, n_{r}$, formed in the vicinity of a small group of mutants (see Appendix $C$ for details). The phenomenological parameter $z$ enables us to tune the direct effect that mutants have on their local N-environment. Using this approach, we consider three scenarios, the first setting a baseline:

(i) Tannin phenotype evolving in the presence of herbivores, with global soil-plant feedbacks, but modelled without taking into account spatial structure.

(ii) Tannin phenotype evolving without herbivory pressure and with local soil-plant feedback, modelled by implicitly taking into account spatial structure.

(iii) Tannin phenotype evolving in the presence of herbivores and with local soil-plant feedback, modelled by implicitly taking into account spatial structure.

In a second step we test the predictions of the first step using simulations of the cellular automaton. We then use the cellular automaton to analyze the "social" effects allowed by spatial structure, such as altruism (van Baalen and Rand, 1998) and ecological inheritance (Odling-Smee et al., 2003). In other words, after analyzing the consequences of a local modification of the environment by tannins (as in the implicit approach), we explored the underlying spatial mechanisms.

In a third step, we relax the assumption that all plants have a fixed symbiotic capacity. 

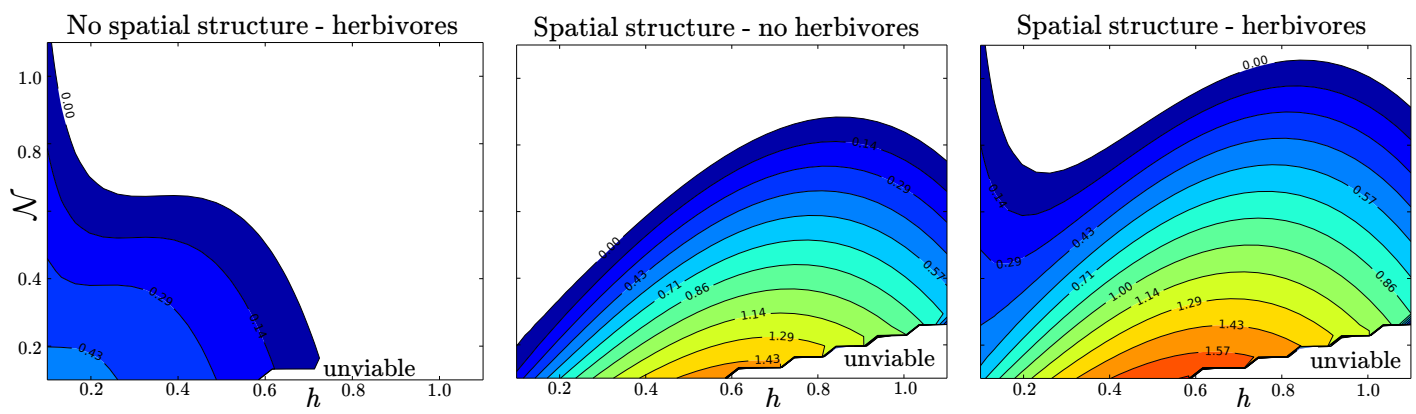

Figure 3: Role of turnover rate $h$ and effective soil fertility $\mathcal{N}$ on the invasibility of tannins. We plotted the fitness gradient at $\tau=0$, a positive value predicts that tannin-producing plants should be selected for. In all panels, the facts that the limits of viability (shaded gray) increases in steps is a numerical artifact. Left panel: non spatial model $(z=0)$ with herbivory $\left(H=10^{-2}\right)$. In this case, recalcitrant $\mathrm{N}$ play no role in the invasion fitness of tannins. middle panel: Spatially implicit context without herbivory $(z=0.03)$. Right panel: spatial structure and herbivory.

Using the spatially implicit version of the model, we sketch the coevolutionary dynamics of tannins and symbiotic capacity. These dynamics follow the fitness gradient, but the latter has now two components: one in the direction of tannin production, and one in the direction of symbiotic capacity. Coevolutionary trajectories follow the fitness gradient until a steady state is reached (Leimar, 2009). For the evolution of single traits, such steady states are called Continuously Stable Strategies (CSS) (Eshel, 1983; Geritz et al., 1998), and co-CSS in a coevolutionary context. In particular, we considered the case where herbivory favors tannin production but where symbiotic capacity cannot evolve without tannins. We asked whether the evolution of tannins as an anti-herbivore defense can then promote the evolution of symbiotic capacity and then further promote tannin production.

A detailed description of those three steps is presented in Appendix C. 


\section{Results}

\section{First step: The spatially implicit approach predicts that infertile environment and intermediate turnover favor tannin evolution}

The relationship between tannin invasibility, soil fertility and plants life strategies under the three scenarios is illustrated in Fig. 3, in which we numerically computed the fitness gradient (eq. 8) as a function of the plant relative turnover rate, $h$, and the effective bare soil $\mathrm{N}$ content, $\mathcal{N}$. In the leftmost panel - i.e. with herbivores but no spatial PSF - we observe selective pressures towards protection against herbivores by tannin production at low turnover rates $h$ and relatively low fertility $\mathcal{N}$. In the middle panel - i.e. with no herbivores but spatial structure - we observe that local recycling of recalcitrant $\mathrm{N}$ by tannin-producing mutants strongly promotes their settlement at low fertility and intermediate turnover rate values. Finally, the rightmost panel - i.e. with both herbivores and spatial structure - combines the previously described behaviors, showing that, for low soil fertility, tannin production can be beneficial at both low and intermediate turnover rate values, but for essentially different reasons. At low turnover rates, tannins are mostly selected for their herbivore deterrent properties, whereas at intermediate turnover rates, they mostly favor plants by forming a local N pool less subject to leaching (see Appendix D for a mathematical derivation of the results presented in Fig. 3).

\section{Second step: simulations of the cellular automaton confirm the predictions of the spatially-implicit approach and demonstrate the importance of social effects}

We confronted the qualitative predictions of the implicit approach to simulations of the cellular automaton, for scenario (ii) i.e. no herbivory (Fig. 4). For the same values of parameters as in Fig 3, we ran simulations of invasion attempts of a tannin producing population $(\tau=0.2)$, starting from a few aggregated individuals in a landscape populated 
Proportion of tannin phenotype at end of simulation

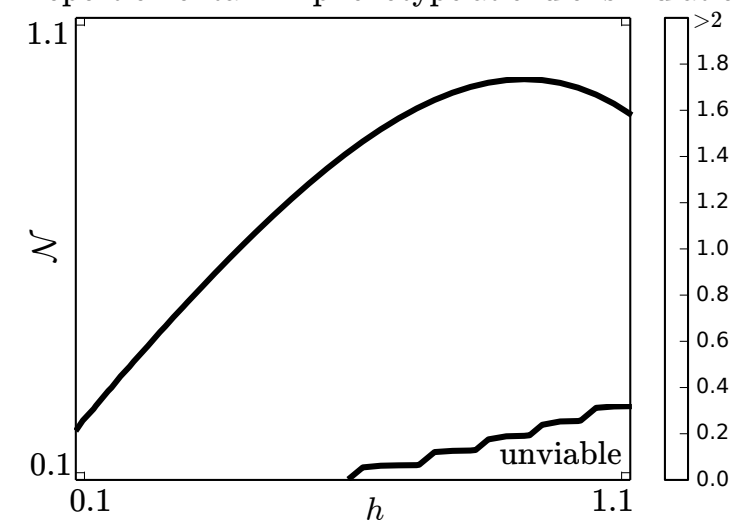

Figure 4: Confrontation of the qualitative predictions of fig.(3) to simulations of the cellular automaton. Each cell represents the ultimate fraction of mutants (with $\tau=0.2$ ) after 400 time steps. The landscape is a periodic lattice comprised of $S=250$ cells. We start from 5 spatially aggregated mutants in a landscape populated by tannin-less plants. New-born individuals have an initial biomass $10^{-3}$ smaller than the one of an average resident. N-diffusion rates are set to $\mathcal{D}_{l}=5$ and $\mathcal{D}_{r}=0.1$. The invasibility domain from the middle panel of Fig. 3 is reported as a black line $(z=0.03)$. Except near the unviable domain where less successful invasions are observed than expected, we observe a good qualitative agreement between the prediction of the implicit approach and simulations. 

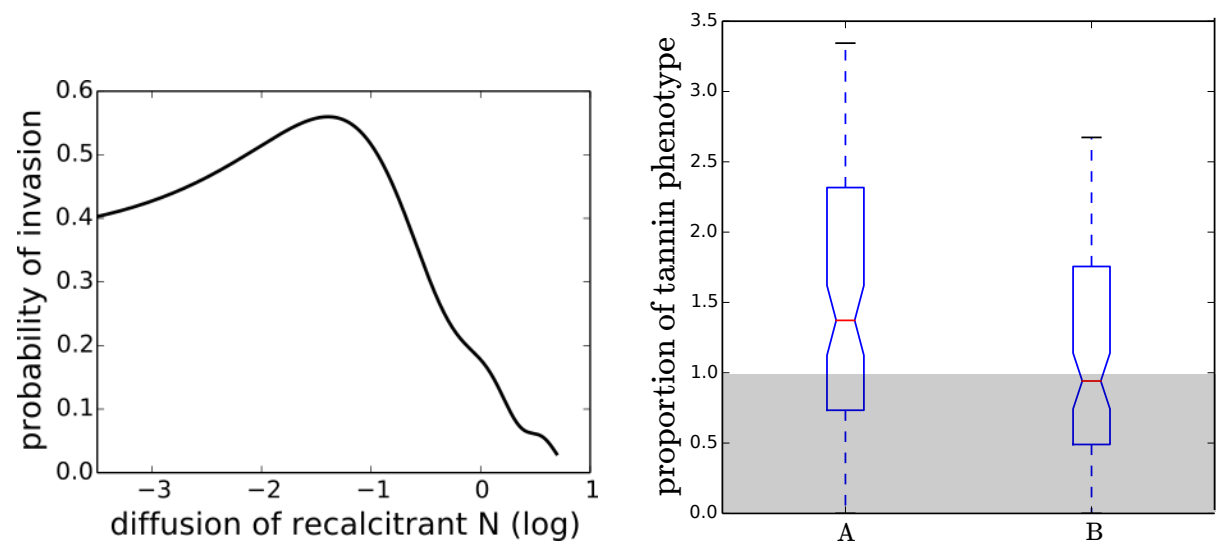

Figure 5: Social effects promoting tannins (simulations of the cellular automaton) Left: Altruism. We varied the recalcitrant diffusion rate $\mathcal{D}_{r}$ from 0 to 2 (shown in log scale) and estimated tannin invasion probability (via the histogram of $\mathcal{D}_{r}$ values for which the ultimate proportion of mutant biomass was larger than one). This probability is not maximal at $\mathcal{D}_{r}=0$, although it should be most beneficial from an individual's perspective (a "selfish" strategy). In terms of probability of invasion, intermediate values of diffusion are more beneficial to tannin-producing plants. Right: ecological inheritance. Box plots are outcomes of invasion attempts (100 runs up to $t=200$ ) A: Same setting as in the left panel, but with $\mathcal{D}_{r}=0$ (so that we can fully control for ecological inheritance in case B). B: When an individual died, the local recalcitrant pool that it formed during its lifetime is deleted (or equivalently, uniformly redistributed), thus removing any effect of ecological inheritance (which is possible because $\mathcal{D}_{r}=0$ ). Comparison between A and $\mathrm{B}$ demonstrates a positive effect of ecological inheritance on the invasion of the tannin phenotype. 
by tannin-less plants. We monitored the ultimate fraction of mutants and compared the outcome of these simulations to the invasibility domain from the middle panel of Fig 3 . Except near the unviable domain where less successful invasions were recorded than expected, we see that simulations of the cellular automaton are in qualitative agreement with the invasibility predictions.

Beyond corroborating the analysis from the spatially-implicit approach, simulations of the cellular automaton allowed us to relate tannin evolution to the evolution of altruism by group selection (van Baalen and Rand, 1998). In the left panel of Fig 5, we plotted the probability of a successful invasion as a function of the recalcitrant diffusion rate $\mathcal{D}_{r}$. At the individual level, in terms of survival and birth rate, the least diffusion of recalcitrant $\mathrm{N}$ the better, i.e. a selfish "behavior". Yet at the group level, in terms of probability of invasion, we observe that intermediate values of diffusion - i.e. altruistic behaviors - are more beneficial. For larger values of nutrient diffusion "cheaters" benefit without costs from the complexed $\mathrm{N}$ pool, and individuals contributing to this pool, having no extra benefits, are eventually excluded.

The death of a tannin producing individual leaves a N-rich patch available for recruitment from neighbors that are likely to be of his kin, a property termed 'ecological inheritance' (Odling-Smee et al., 2003). As illustrated in Fig 5, this effect was positive in our model: without ecological inheritance (case B in Fig 5) the invasion is substantially slower.

\section{Third step: positive coevolutionary feedbacks between tannins and symbiotic capacity can be triggered by herbivores}

In Fig. 6, using the spatially implicit version of the model, we sketch the coevolutionary dynamics in trait space $(e, \tau)$, generated by the fitness gradient (Leimar, 2009). The coCSS (i.e. the evolutionary steady-state of the two traits) corresponds to an intersection between the fitness gradient isocline of symbiotic capacity (dashed line in Fig. 6) and 


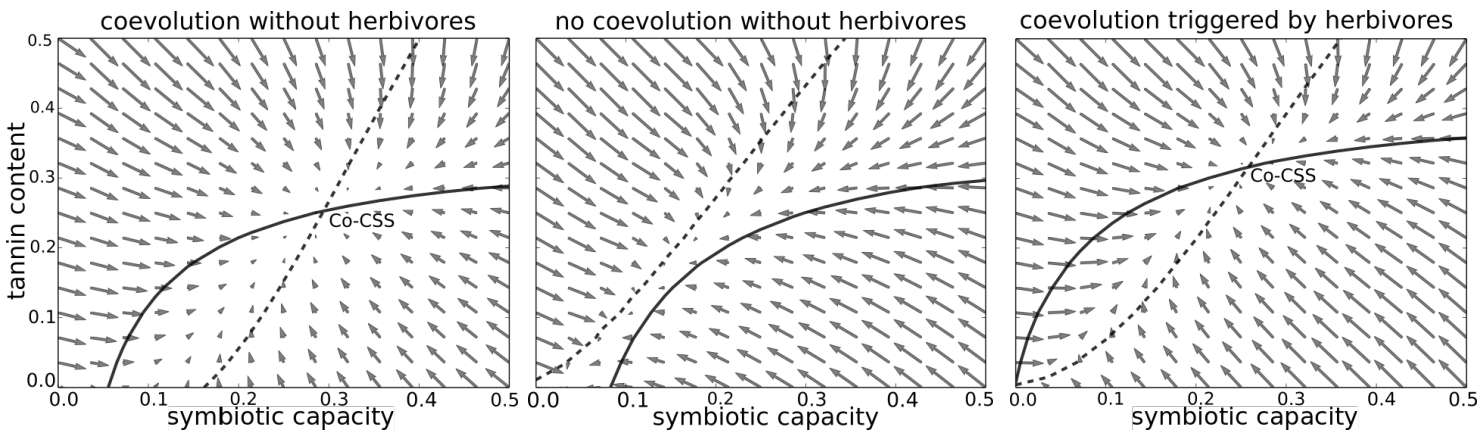

Figure 6: Coevolution of tannins and symbiotic capacity. The dashed line is the fitness gradient isocline of symbiotic capacity. The black line is the fitness gradient isocline of the tannin phenotype. The coevolutionary Continously Stable Strategy (co-CSS) is at the intersection of the two. Leftmost panel: we chose parameters of absorption efficiencies so that that symbiotic capacity would be favored even in the absence of tannins. In this case, we predict a coevolutionary positive feedback, with tannin evolution increasing the selective advantage of symbiotic associations and vice versa, until a co-CSS is reached. Middle panel: here we chose absorption efficiencies so that symbiotic capacity is not favored in the absence of tannins. In this case, we predict no evolution of tannin production nor symbiotic capacity. Rightmost panel: Additional pressure from herbivory, however, can trigger a coevolutionary positive feedback. 
the one of tannin content (black line in Fig. 6). In the first panel, labile absorption is sufficiently inefficient that symbiotic capacity can be selected even in the absence of recalcitrant $\mathrm{N}$ (the fitness gradient along $e$ is positive at $e, \tau=0$ ). We observe a positive coevolutionary feedback: if symbiotic capacity increases, complexed $\mathrm{N}$ can be absorbed so that tannins can become beneficial, which in turn enhances the selective pressure on symbiotic capacity, and so on until the co-CSS is reached. In the middle panel, symbiotic capacity is too costly to evolve in the absence of tannins, and no coevolution is possible. However, in the rightmost panel, herbivory allows the evolution of tannins as an antiherbivore defense. This triggers a positive coevolutionary feedback: symbiotic capacity evolves which can enhance tannins until the co-CSS is reached. This demonstrates the potential complementary of the two perspectives concerning tannin evolution in plants (i.e. anti-herbivore defense and positive plant soil feedbacks): the fact that tannins form anti-herbivore compounds can be at the origin of the PSF that can, in turn, promote tannin production and mycorrhizal associations.

\section{Discussion}

\section{Positive plant soil feedbacks alone can trigger tannin evolution}

Our results support hypothesis 1, and are in line with the "litter perspective" proposed by Hättenschwiler et al. (2011) as an alternative to the "green foliage perspective" for the evolution of tannins. When associated with symbionts that allow them to retrieve nutrients directly from recalcitrant organic matter, plants benefit from the afterlife effects of tannins, instead of suffering from a reduction of nutrient cycling (Barot et al., 2014). In line with empirical findings, our model assumes that the main mechanism explaining the impact of tannins on the soil is their ability to complex proteins (Joanisse et al., 2009; Kraus et al., 2004), thus forming a recalcitrant $N$ pool that can be reabsorbed by mycorrhizae (Wurzburger and Hendrick, 2009). 
This can confer a selective advantage to tannin-producing plants with intermediate plant tissue turnover rates (Figs 3-4). If plant tissue turnover rate is too slow, plant biomass accumulates, photosynthesis becomes limited by intraspecific competition and the carbon stock is too low for plants to invest in tannins. By contrast, if turnover is too fast, labile nutrients abound and the recalcitrant pool formed by tannins becomes useless. Importantly, tannin-producing plants must have a preferential access to the recalcitrant pool. The mechanisms underlying such exclusivity may be spatial (as in our model), or biological, if mycorrhizae are better adapted to reabsorb the recalcitrant nutrients formed by their host (Joanisse et al., 2009; Wu et al., 2003; Wurzburger and Hendrick, 2009). Tannin production cannot evolve when the recalcitrant pool is shared by all plants, because tannin producing plants must be protected from the invasion of cheaters able to absorb recalcitrant $\mathrm{N}$ without paying the cost of tannin production.

Our model is in this sense similar to those focused on the evolution of altruism (Le Galliard et al., 2003; van Baalen and Rand, 1998), facilitation (Kéfi et al., 2008) and shared chemical resources among microorganisms (Allen et al., 2013). Yet our model is not a mere illustration of altruism evolution. Indeed, in models of altruism evolution, the altruistic trait is beneficial to any phenotype (Wilson, 1980). Our model is one of competition for resources in which no individual is strictly altruistic. In principle, tannin production could both lead to the competitive exclusion of non-symbiotic plants (Joanisse et al., 2009) and to the facilitation of plants associated with mycorrhizae. Furthermore, we considered here a whole ecosystem including abiotic compartments, instead of a single monospecific population. Hence, selection does not operate at the group-level (van Baalen and Rand, 1998), but at the ecosystem-level (see Tannin evolution under a niche-construction perspective, below). 


\section{Nutrient-poor ecosystems favor tannin evolution}

Because they slow down the mineralization of recycled nutrients, one could have expected tannins to be detrimental to plants in infertile ecosystems (Barot et al., 2014). This prediction, however, is not verified empirically, both at the inter- and intraspecific level: tannin producing species are mostly found in infertile environments (Endara and Coley, 2011; McKey et al., 1978), and plant populations of a single species contain more tannin in nutrient-poor environments (Hofland-Zijlstra and Berendse, 2009; Kouki and Manetas, 2002; Kraus et al., 2004; Northup et al., 1995). In line with hypothesis 2, our model resolves this apparent paradox (Fig. 3, middle panel). Tannins can be beneficial to plants growing in infertile ecosystems because they allow the formation of recalcitrant nutrient

pools less prone to leaching (Northup et al., 1998), which can counter-balance the negative effect of a slower mineralization (Schweitzer et al., 2008). In the model of Barot et al. (2014), the nutrients present in dead organic matter could not be reabsorbed by plants directly, and had to be first decomposed into mineral nutrients, leading to the conclusion that plants must evolve fast mineralization strategies in infertile ecosystems. By contrast, in our model plants have direct access to the recalcitrant pool via symbiotic associations with mycorrhizal fungi. In fact, our results suggest that fertile ecosystems prevent tannin evolution. In our model, the positive PSF initiated by tannins were inefficient at high fertility. Furthermore, fertile ecosystems favor fast growing species with high turnover rates (Endara and Coley, 2011), which may weaken the selective pressure towards tannin production (leftmost panel of Fig. 3).

\section{The importance of symbiosis with mycorrhizae}

In line with hypothesis 3, our model predicts non-trivial coevolutionary dynamics that can lead to an equilibrium strategy in which tannin production favors the association with mycorrhizal fungi and vice-versa (Fig 6). If plants benefit from a symbiotic association even when recalcitrant nutrients are absent, this equilibrium can be reached without 
any defensive role of tannins against herbivory (Fig 6, leftmost panel). If plants do not require symbiotic associations to absorb labile nutrients, a defensive role of tannins against herbivory, causing a build-up of recalcitrant nutrients inaccessible without symbiotic associations, can trigger the coevolutionary dynamics (Fig 6 rightmost panels). Hence, herbivory and positive plant soil feedbacks may act synergistically on tannin evolution. This assertion, however, calls for a cautionary remark. Indeed, our model assumes that herbivory is equivalent to biomass turnover, without impacting nutrient cycling (Belovsky and Slade, 2000). However, Kagata and Ohgushi (2013) found that plant litter led to positive PSF, showcasing a "home-field advantage", whereas insect frass did not. Therefore, herbivory might facilitate tannin evolution through the "green foliage perspective", and simultaneously weaken (or even cancel) the positive PSF of the "litter perspective".

Our results and the above reasoning all posit the ability of mycorrhizae to access nitrogen from tannin-protein complexes. Within functional groups of mycorrhizae, the mycorrhizal strategy may have evolved several times from free-living ancestors (Pellitier and Zak, 2018). The different groups of mycorrhizae are not phylogenetically related and have distinct enzymatic capacity (Phillips et al., 2013). Consequently, the decomposer capacities of mycorrhizae depend on their type - ectomycorrhizae (ECM), ericoidmycorrhizae (ERM), and endomycorrhizae (AMF) - and of the clade within a given type.

That mycorrhizae can access nitrogen from recalcitrant complexes is still in debate for ECM (Lindahl and Tunlid, 2015; Pellitier and Zak, 2018), the dominant type in temperate and boreal forests. The loss of genes associated with saprotrophic ability during the transition from a free to a mycorrhizal strategy, as well as experimental evidence (Bending and Read, 1996), suggest a low ability for complex organic matter decomposition. Yet, an increasing body of literature suggests the opposite, arguing that ECM selectively mine complex organic nitrogen (Averill and Hawkes, 2016; Shah et al., 2016; Trap et al., 2017). Wurzburger and Hendrick (2009), using 15 N-enriched protein-tannin complexes from leaf litter extracts, convincingly demonstrated the ability of ERM to acquire nitrogen 
from tannin-protein complexes (ERM abound in heathland and peatlands which often harbour tannin-rich plants). AMF, which dominate in grasslands and tropical forests, are usually poor decomposers, and may not be able to acquire nitrogen from tanninprotein complexes. However, as pointed by Hättenschwiler et al. (2011), this ability has yet to be tested for tropical AM fungi. Furthermore, AMF create positive PSF which can counteract the Janzen-Connell effects of soil pathogens (Liang et al., 2015).

In short, if our results are relevant to ERM dominated ecosystems (e.g. heathland and peatlands), more studies are needed to clarify the decomposer ability of ECM and tropical AM fungi, and thus establish the precise level of generality of our results.

Incidentally, our study sheds light on the mycorrhizal-associated nutrient economy framework proposed by Phillips et al. (2013). This framework posits that the contrasting modes of nutrient acquisition of different mycorrhizal fungi can explain contrasting $\mathrm{C}$ and $\mathrm{N}$ cycling patterns observed in ecosystems dominated by ECM, ERM or AM plants. By modeling together the formation of recalcitrant $\mathrm{N}$ through tannin production and the ability of mycorrhizae to access it, our study refines this framework and places the patterns observed by Phillips et al. (2013) in an evolutionary perspective.

\section{Tannin evolution under a niche-construction perspective}

The evolution of tannins through positive plant soil feedbacks can be understood as a case of niche construction, i.e. organisms' ability to modify their environment for their own benefit, or for the benefit of related organisms which will occupy the improved environment after their death (Kylafis and Loreau, 2008; Lehmann, 2008; Odling-Smee et al., 2003). In our model tannins initiate an eco-evolutionary loop between organisms and their environments. The death of a tannin producing individual leaves a N-rich patch available for recruitment from neighbors that are likely to be of his kin. Individuals could inherit the environment transformed by their ancestors, a property termed ecological inheritance (Odling-Smee et al., 2003). As illustrated in Fig 5, this effect was 
substantial and positive in our model. Importantly, this niche constructing mechanism only relies on a degree of locality of the ecosystem dynamics (Zelnik et al., 2019), thus only requires that the dispersal of organisms and diffusion of nutrients across patches are not too strong. Most evolutionary models consider the evolution of phenotypes whose fitness can be fully understood at the organismic level. In the case of tannin evolution, the fitness of this phenotype can be understood at the organismic level in the presence of herbivores, but not in the presence of the positive PSF. The feedback involves plants, nutrient compartments, and mycorrhizae within the soil, and competition occurs between ecosystems having contrasted material cycles (Loreau, 1998, 2010). A whole ecosystem therefore persists through time via genetic and ecological inheritance. Within the framework of multi-level selection (Okasha, 2006), we therefore suggest that tannin production evolved as the result of ecosystem-level selection (Goodnight, 2000; Swenson et al., 2000).

\section{Conclusion}

(i) Our model shows that the protective role of tannins against herbivores reinforces their evolution but is not a necessary condition. Positive PSF can be strong enough to trigger alone tannin evolution (in line with hypothesis 1), provided that the pool of recalcitrant nutrients complexed by tannins is, at least in part, reabsorbed by the mycorrhizae of the same plant, or by its neighboring relatives (justified by hypothesis 3). We therefore propose that niche construction through positive PSF can play a decisive role in the evolution of tannin production, in consonance with empirical studies (Hättenschwiler et al., 2011; Schweitzer et al., 2008).

(ii) Our model explains why tannin evolution is more likely in infertile ecosystems, in agreement with empirical findings (Endara and Coley, 2011; McKey et al., 1978). This is a key point as it was previously unclear how tannin evolution was modulated by ecological conditions. According to our model, tannin evolution also requires plants to have intermediate biomass turnover rates. Given that fertile ecosystems tend to harbor 
plants with high turnover (Endara and Coley, 2011), this further highlights the fact that tannin evolution is more effective in nutrient-limited ecosystems.

(iii) More generally, our work provides a detailed example of how the evolution of niche constructing traits can depend on large-scale ecosystem properties such as fertility, bridging the gap between ecology and evolution.

\section{Acknowledgments}

We thank Christiane Gallet for her helpful advices on an earlier version of this manuscript, and Matthieu Barbier for his help in programming the cellular automaton.

\section{Authors' contributions}

JFA, SC, SK, and SI conceived the ideas and designed methodology; JFA designed and analysed the mathematical model and wrote the code. JFA and SI led the writing of the manuscript. All authors contributed critically to the drafts and gave final approval for publication.

\section{Data accessibility}

Python code was written and used to generate the results presented in figures 2-6. (available on DRYAD).

\section{References}

Aerts, R., and F. Chapin. 2000. The mineral nutrition of wild plants revisited: A reevaluation of processes and patterns. Pages 1-67 in Advances in Ecological Research, Vol 30, Advances in Ecological Research. Academic Press Inc, San Diego. 
Allen, B., J. Gore, and M. A. Nowak. 2013. Spatial dilemmas of diffusible public goods. eLife 2:e01169.

Averill, C., and C. V. Hawkes. 2016. Ectomycorrhizal fungi slow soil carbon cycling. Ecology letters 19:937-947.

Ayres, E., H. Steltzer, B. L. Simmons, R. T. Simpson, J. M. Steinweg, M. D. Wallenstein, N. Mellor, W. J. Parton, J. C. Moore, and D. H. Wall. 2009. Home-field advantage accelerates leaf litter decomposition in forests. Soil Biology and Biochemistry 41:606610.

Barot, S., S. Bornhofen, N. Loeuille, N. Perveen, T. Shahzad, and S. Fontaine. 2014. Nutrient enrichment and local competition influence the evolution of plant mineralization strategy: a modelling approach. Journal of Ecology 102:357-366.

Bate-Smith, E., and T. Swain. 1962. Flavonoid compounds. Pages 755-809 in H. Mason and A. Florkin, eds. Comparative Biochemistry. Academic Press, New York.

Belovsky, G. E., and J. B. Slade. 2000. Insect herbivory accelerates nutrient cycling and increases plant production. Proceedings of the National Academy of Sciences 97:1441214417.

Bending, G., and D. Read. 1996. Nitrogen mobilization from protein-polyphenol complex by ericoid and ectomycorrhizal fungi. Soil Biology \& Biochemistry 28:1603-1612.

Benoit, R., and R. Starkey. 1968. Enzyme inactivation as a factor in the inhibition of decomposition of organic matter by tannins. Soil Science 105:203-208.

Bergvall, U. A., and O. Leimar. 2005. Plant secondary compounds and the frequency of food types affect food choice by mammalian herbivores. Ecology 86:2450-2460.

Bernays, E. A., G. C. Driver, and M. Bilgener. 1989. Herbivores and plant tannins. Pages 263-302 in Advances in ecological research, vol. 19. Elsevier. 
Coq, S., J. Souquet, E. Meudec, V. Cheynier, and S. Hattenschwiler. 2010. Interspecific variation in leaf litter tannins drives decomposition in a tropical rain forest of French Guiana. Ecology 91:2080-2091.

Danger, M., T. Daufresne, F. Lucas, S. Pissard, and G. Lacroix. 2008. Does Liebig's law of the minimum scale up from species to communities? Oikos 117:1741-1751.

Donohue, K., L. Dorn, C. Griffith, E. Kim, A. Aguilera, C. R. Polisetty, and J. Schmitt. 2005. Niche construction through germination cueing: life-history responses to timing of germination in Arabidopsis thaliana. Evolution 59:771-785.

Droop, M. R. 1968. The kinetics of uptake, growth and inhibition in Monochrysis lutheri. Journal of the Marine Biological Association of the United Kingdom 48:689-733.

Endara, M., and P. Coley. 2011. The resource availability hypothesis revisited: a metaanalysis. Functional Ecology 25:389-398.

Eshel, I. 1983. Evolutionary and continuous stability. Journal of theoretical Biology 103:99-111.

Feeny, P. 1970. Seasonal Changes in Oak Leaf Tannins and Nutrients as a Cause of Spring Feeding by Wintr Moth Caterpillars. Ecology 51:565-581.

Geritz, S. A., J. A. Metz, E. Kisdi, and G. Meszéna. 1997. Dynamics of adaptation and evolutionary branching. Physical Review Letters 78:2024.

Geritz, S. a. H., E. Kisdi, G. Meszéna, and J. a. J. Metz. 1998. Evolutionarily singular strategies and the adaptive growth and branching of the evolutionary tree. Evolutionary Ecology 12:35-57.

Goodnight, C. J. 2000. Heritability at the ecosystem level. Proceedings of the National Academy of Sciences 97:9365-9366. 
Govaert, L., E. A. Fronhofer, S. Lion, C. Eizaguirre, D. Bonte, M. Egas, A. P. Hendry, A. De Brito Martins, C. J. Melián, and J. A. Raeymaekers. 2019. Eco-evolutionary feedbacks-Theoretical models and perspectives. Functional Ecology 33:13-30.

Hagerman, A. E., and L. G. Butler. 1991. Tannins and lignins. In 'Herbivores: Their Interactions with Secondary Plant Metabolites'.(Eds GA Rosenthal and MR Berenbaum.) pp. 355-388. Academic Press: San Diego.

Hättenschwiler, S., and P. Vitousek. 2000. The role of polyphenols in terrestrial ecosystem nutrient cycling. Trends in Ecology \& Evolution 15:238-243.

Hofland-Zijlstra, J. D., and F. Berendse. 2009. The effect of nutrient supply and light intensity on tannins and mycorrhizal colonisation in Dutch heathland ecosystems. Plant Ecology 201:661-675.

Hättenschwiler, S., S. Coq, S. Barantal, and I. T. Handa. 2011. Leaf traits and decomposition in tropical rainforests: revisiting some commonly held views and towards a new hypothesis. New Phytologist 189:950-965.

Joanisse, G., R. Bradley, C. Preston, and A. Munson. 2007. Soil enzyme inhibition by condensed litter tannins may drive ecosystem structure and processes: the case of Kalmia angustifolia. New Phytologist 175:535-546.

Joanisse, G. D., R. L. Bradley, C. M. Preston, and G. D. Bending. 2009. Sequestration of soil nitrogen as tannin-protein complexes may improve the competitive ability of sheep laurel (Kalmia angustifolia) relative to black spruce (Picea mariana). New Phytologist 181:187-198.

Jordan, C., R. Todd, and G. Escalante. 1979. Nitrogen Conservation in a Tropical Rain Forest. Oecologia 39:123-128. 
Kagata, H., and T. Ohgushi. 2013. Home-field advantage in decomposition of leaf litter and insect frass. Population ecology 55:69-76.

Klironomos, J. N. 2002. Feedback with soil biota contributes to plant rarity and invasiveness in communities. Nature 417:67.

Kouki, M., and Y. Manetas. 2002. Resource availability affects differentially the levels of gallotannins and condensed tannins in Ceratonia siliqua. Biochemical Systematics and Ecology 30:631-639.

Kraus, T., R. Zasoski, and R. Dahlgren. 2004. Fertility and pH effects on polyphenol and condensed tannin concentrations in foliage and roots. Plant and Soil 262:95-109.

Kraus, T. E. C., R. A. Dahlgren, and R. J. Zasoski. 2003. Tannins in nutrient dynamics of forest ecosystems - a review. Plant and Soil 256:41-66.

Kylafis, G., and M. Loreau. 2008. Ecological and evolutionary consequences of niche construction for its agent. Ecology letters 11:1072-1081.

Kéfi, S., M. v. Baalen, M. Rietkerk, and M. Loreau. 2008. Evolution of local facilitation in arid ecosystems. The American Naturalist 172:E1-E17.

Laland, K. N., F. J. Odling-Smee, and M. W. Feldman. 1999. Evolutionary consequences of niche construction and their implications for ecology. Proceedings of the National Academy of Sciences 96:10242-10247.

Le Galliard, J.-F., R. Ferriére, and U. Dieckmann. 2003. The Adaptive Dynamics of Altruism in Spatially Heterogeneous Populations. Evolution 57:1-17.

Lehmann, L. 2008. The adaptive dynamics of niche constructing traits in spatially subdivided populations: evolving posthumous extended phenotypes. Evolution 62:549-566.

Leimar, O. 2009. Multidimensional convergence stability. Evolutionary Ecology Research 11:191-208. 
Lemesle, V., and L. Mailleret. 2008. A mechanistic investigation of the algae growth “Droop" model. Acta biotheoretica 56:87-102.

Lewontin, R. C. 1983. The Organism as the Subject and Object of Evolution. Scientia 77:65.

Liang, M., X. Liu, R. S. Etienne, F. Huang, Y. Wang, and S. Yu. 2015. Arbuscular mycorrhizal fungi counteract the Janzen-Connell effect of soil pathogens. Ecology 96:562-574.

Lindahl, B. D., and A. Tunlid. 2015. Ectomycorrhizal fungi-potential organic matter decomposers, yet not saprotrophs. New Phytologist 205:1443-1447.

Loreau, M. 1998. Ecosystem development explained by competition within and between material cycles. Proceedings of the Royal Society of London B: Biological Sciences 265:33-38.

- 2010. Evolution of ecosystems and ecosystem properties. Pages 225-259 in From Populations to Ecosystems, Theoretical Foundations for a New Ecological Synthesis. Princeton University Press.

McKey, D., G. Peter, C. Mbi, J. Gartlan, and T. Struhsaker. 1978. Phenolic Content of Vegetation in Two African Rain Forests: Ecological Implications. Science 202:61-64.

Mole, S. 1993. The systematic distribution of tannins in the leaves of angiosperms: A tool for ecological studies. Biochemical Systematics and Ecology 21:833-846.

Northup, R., Z. Yu, R. Dahlgren, and K. Vogt. 1995. Polyphenol Control of Nitrogen Release from Pine Litter. Nature 377:227-229.

Northup, R. R., R. A. Dahlgren, and J. G. McColl. 1998. Polyphenols as regulators of plant-litter-soil interactions in northern California's pygmy forest: a positive feedback? Pages 189-220 in Plant-induced soil changes: Processes and feedbacks. Springer. 
Odling-Smee, F. J., K. N. Laland, and M. W. Feldman. 2003. Niche construction: the neglected process in evolution. 37. Princeton University Press.

Odum, E. P. 1971. Fundamentals of ecology. 3rd ed. Saunders, Philadelphia.

Okasha, S. 2006. Evolution and the Levels of Selection, vol. 16. Clarendon Press Oxford.

Ordoñez, J. C., P. M. Van Bodegom, J.-P. M. Witte, I. J. Wright, P. B. Reich, and R. Aerts. 2009. A global study of relationships between leaf traits, climate and soil measures of nutrient fertility. Global Ecology and Biogeography 18:137-149.

Pellitier, P. T., and D. R. Zak. 2018. Ectomycorrhizal fungi and the enzymatic liberation of nitrogen from soil organic matter: why evolutionary history matters. New Phytologist 217:68-73.

Phillips, R. P., E. Brzostek, and M. G. Midgley. 2013. The mycorrhizal-associated nutrient economy: a new framework for predicting carbon-nutrient couplings in temperate forests. New Phytologist 199:41-51.

Pritsch, K., and J. Garbaye. 2011. Enzyme secretion by ECM fungi and exploitation of mineral nutrients from soil organic matter. Annals of Forest Science 68:25-32.

Putten, W. H. v. d., R. D. Bardgett, J. D. Bever, T. M. Bezemer, B. B. Casper, T. Fukami, P. Kardol, J. N. Klironomos, A. Kulmatiski, J. A. Schweitzer, K. N. Suding, T. F. J. V. d. Voorde, and D. A. Wardle. 2013. Plant-soil feedbacks: the past, the present and future challenges. Journal of Ecology 101:265-276.

Read, D., and J. Perez-Moreno. 2003. Mycorrhizas and nutrient cycling in ecosystems - a journey towards relevance? New Phytologist 157:475-492.

Schweitzer, J., J. Bailey, B. Rehill, G. Martinsen, S. Hart, R. Lindroth, P. Keim, and T. Whitham. 2004. Genetically based trait in a dominant tree affects ecosystem processes. Ecology Letters 7:127-134. 
Schweitzer, J., I. Juric, T. Voorde, K. Clay, W. Putten, and J. Bailey. 2014. Are there evolutionary consequences of plant-soil feedbacks along soil gradients? Functional Ecology 28:55-64.

Schweitzer, J. A., M. D. Madritch, J. K. Bailey, C. J. LeRoy, D. G. Fischer, B. J. Rehill, R. L. Lindroth, A. E. Hagerman, S. C. Wooley, and S. C. Hart. 2008. From genes to ecosystems: the genetic basis of condensed tannins and their role in nutrient regulation in a Populus model system. Ecosystems 11:1005-1020.

Schwilk, D. W. 2003. Flammability is a niche construction trait: canopy architecture affects fire intensity. The American Naturalist 162:725-733.

Shah, F., C. Nicolás, J. Bentzer, M. Ellström, M. Smits, F. Rineau, B. Canbäck, D. Floudas, R. Carleer, and G. Lackner. 2016. Ectomycorrhizal fungi decompose soil organic matter using oxidative mechanisms adapted from saprotrophic ancestors. New Phytologist 209:1705-1719.

Swenson, W., D. S. Wilson, and R. Elias. 2000. Artificial ecosystem selection. Proceedings of the National Academy of Sciences 97:9110-9114.

Trap, J., M. Akpa-Vinceslas, P. Margerie, S. Boudsocq, F. Richard, T. Decaëns, and M. Aubert. 2017. Slow decomposition of leaf litter from mature Fagus sylvatica trees promotes offspring nitrogen acquisition by interacting with ectomycorrhizal fungi. Journal of Ecology 105:528-539.

van Baalen, M., and D. A. Rand. 1998. The unit of selection in viscous populations and the evolution of altruism. Journal of Theoretical Biology 193:631-648.

Van Nuland, M. E., I. M. Ware, J. K. Bailey, and J. A. Schweitzer. 2019. Ecosystem feedbacks contribute to geographic variation in plant-soil eco-evolutionary dynamics across a fertility gradient. Functional Ecology 33:95-106. 
Van Nuland, M. E., R. C. Wooliver, A. A. Pfennigwerth, Q. D. Read, I. M. Ware, L. Mueller, J. A. Fordyce, J. A. Schweitzer, and J. K. Bailey. 2016. Plant-soil feedbacks: connecting ecosystem ecology and evolution. Functional Ecology 30:1032-1042.

Whitham, T. G., J. K. Bailey, J. A. Schweitzer, S. M. Shuster, R. K. Bangert, C. J. LeRoy, E. V. Lonsdorf, G. J. Allan, S. P. DiFazio, and B. M. Potts. 2006. A framework for community and ecosystem genetics: from genes to ecosystems. Nature Reviews Genetics 7:510523.

Wilson, D. S. 1980. The natural selection of populations and communities. Benjamin/Cummings Pub. Co.

Wu, T., J. N. Sharda, and R. T. Koide. 2003. Exploring interactions between saprotrophic microbes and ectomycorrhizal fungi using a protein-tannin complex as an $\mathrm{N}$ source by red pine (Pinus resinosa). New Phytologist 159:131-139.

Wurzburger, N., and R. Hendrick. 2009. Plant litter chemistry and mycorrhizal roots promote a nitrogen feedback in a temperate forest. Journal of Ecology 97:528-536.

Zelnik, Y. R., J.-F. Arnoldi, and M. Loreau. 2019. The three regimes of spatial recovery. Ecology 100:e02586. 


\section{Online Appendix}

\section{Appendix A: Details of the mean-field model}

\section{Plant growth}

Plant biomass is modeled by its amount of $\mathrm{C}$ and $\mathrm{N}$ content. We denote $B_{\tau}$ the $\mathrm{C}+\mathrm{N}$ content of plant biomass, with C:N ratio $\alpha_{\tau}$. A fraction $\tau$ of plant biomass is used to produce tannins, whereas the rest is involved in all other physiological processes, like growth, nutrient absorption or $\mathrm{C}$ exchange with mycorrhizae. Biomass is therefore constituted by a proportion $\tau B_{\tau}$ of tannin-C, and by the remaining biomass, $B=(1-\tau) B_{\tau}$, referred to as the effective biomass with C:N ratio $\alpha$. We denote $\gamma$ and $\gamma_{\tau}$ the proportion of $\mathrm{N}$ in $B$ and $B_{\tau}$, respectively, with $1-\gamma$ and $1-\gamma_{\tau}$, representing the complementary proportions of $\mathrm{C}$ in those compartments. $\alpha_{\tau}$ and $\gamma_{\tau}$ are simple functions of $\tau$ and $\alpha$,

$$
\gamma_{\tau}=(1-\tau) \gamma, \text { and } \alpha_{\tau}=\frac{\alpha+\tau}{1-\tau}
$$

At any given time, biomass growth is controlled by the limiting factor among $\mathrm{C}$ and $\mathrm{N}$ stocks (denoted $n$ and c), i.e. the minimum between $\frac{n}{\gamma_{\tau}}$ and $\frac{c}{1-\gamma_{\tau}}$ (Danger et al., 2008; Odum, 1971). Under these assumptions, and a turnover rate $h$, the dynamics of plant biomass $B_{\tau}$ follow

$$
\frac{1}{\mu} \frac{d B_{\tau}}{d t}=\underbrace{\min \left\{\frac{n}{\gamma_{\tau}}, \frac{c}{1-\gamma_{\tau}}\right\}}_{\text {growth }}-\underbrace{h B_{\tau}}_{\text {turnover and herbivory }}
$$

Here $\mu$ is a rate, which sets plants' ecological timescale. We also model the effect of herbivory, that decreases plant biomass, as a part of the global turnover rate $h . h$ is a decreasing function of $\tau$ when herbivores are present:

$$
h(\tau)=h(1)+(1-\tau) H
$$

where $(1-\tau) H$ is the relative rate of herbivory, reduced by tannins and reflecting the fact that they act as anti-herbivore compounds. 


\section{Resource acquisition by plants}

In the soil, $\mathrm{N}$ is divided in two compartments: labile $n_{l}$, and recalcitrant $n_{r}$. Recalcitrant $\mathrm{N}$ is produced by plants themselves when tannins complex $\mathrm{N}$-containing proteins during leaf senescence (Northup et al., 1995). Recalcitrant $\mathrm{N}$ is less subject to leaching and volatilization but is more difficult to absorb than labile $\mathrm{N}$ (Jordan et al., 1979). A critical assumption of our model is that symbiotic associations with mycorrhizae are necessary to absorb recalcitrant N (Pritsch and Garbaye, 2011; Read and Perez-Moreno, 2003; Wurzburger and Hendrick, 2009). We model this requirement by writing labile and recalcitrant absorption efficiencies (resp. $\mathcal{E}_{l}$ and $\mathcal{E}_{r}$ ) as explicit functions of $e$ :

$$
\mathcal{E}_{l}(e)=\mathcal{E}_{l}^{-}+e\left(\mathcal{E}_{l}^{+}-\mathcal{E}_{l}^{-}\right) \text {and } \mathcal{E}_{r}(e)=e \mathcal{E}_{r}^{+}
$$

where $\mathcal{E}_{i}^{-}$and $\mathcal{E}_{i}^{+}$denote the extreme efficiency values. Thus, in our model, symbiotic capacity enhances the absorption capacity of both labile and recalcitrant nitrogen, but is necessary to absorb recalcitrant $\mathrm{N}$. Being associated with mycorrhizae comes at a cost of maintenance $m$, linked to symbiotic capacity by

$$
m(e)=\frac{m_{0}}{1-e}
$$

where $m_{0}$ is the baseline maintenance cost. We note $\xi$ the rate at which the absorption process is running, generating a cost $\xi m$. On the other hand, plants access $C$ by photosynthesis, at a rate $\varphi$ which saturates, due to competition for light, as $B_{\tau}$ grows:

$$
\varphi\left(B_{\tau}\right)=\frac{\varphi_{0}}{1+B_{\tau}}
$$

With this equation, $B_{\tau}$ is restricted to values smaller than one, thus defining a mass scale in our model. The presence of $\gamma B$ in the photosynthesis term accounts for the fact that it is mostly N-compounds of biomass that perform photosynthesis. Under these assumptions, $\mathrm{C}$ and $\mathrm{N}$ stocks follow, in physiological time scales $t^{\prime}$, (which we will 
assume much shorter than the ecological time scale)

$$
\begin{aligned}
& \frac{d n}{d t^{\prime}}=\underbrace{\xi \sum_{i=l, r} \mathcal{E}_{i} n_{i} B}_{\text {absorption }}-\gamma_{\tau} \underbrace{\min \left\{\frac{n}{\gamma_{\tau}}, \frac{c}{1-\gamma_{\tau}}\right\}}_{\text {growth }}-\underbrace{h n}_{\text {turnover }} \\
& \frac{d c}{d t^{\prime}}=\underbrace{\varphi \gamma B}_{\text {photosynthesis }}-\underbrace{\xi m B}_{\text {maintenance }}-\left(1-\gamma_{\tau}\right) \underbrace{\min \left\{\frac{n}{\gamma_{\tau}}, \frac{c}{1-\gamma_{\tau}}\right\}}_{\text {growth }}-\underbrace{h c}_{\text {turnover }}
\end{aligned}
$$

Physiological dynamics are best expressed in terms of concentrations $p=n / B$ and $q=$ $c / B$ (Lemesle and Mailleret, 2008). Using $\frac{d p}{d t^{\prime}}=\frac{d n}{d t^{\prime}} \frac{1}{B}-\frac{d B}{d t^{\prime}} \frac{n}{B^{2}}$ the above system becomes:

$$
\left\{\begin{array}{l}
\frac{d p}{d t^{\prime}}=\xi \sum_{i=l, r} \mathcal{E}_{i} n_{i}-(p+\gamma) \kappa(p, q) \\
\frac{d q}{d t^{\prime}}=\gamma \varphi-m \xi-(q+1-\gamma) \kappa(p, q)
\end{array}\right.
$$

where $\kappa(p, q)=(1-\tau) \min \left\{\frac{p}{\gamma_{\tau}}, \frac{q}{1-\gamma_{\tau}}\right\}$. We assume physiological dynamics to rapidly reach a moving equilibrium $\left(p_{*}, q_{*}\right)$ defined by $\left.\frac{d p}{d t^{\prime}}\right|_{\left(p_{*}, q_{*}\right)}=\left.\frac{d q}{d t^{\prime}}\right|_{\left(p_{*}, q_{*}\right)}=0$. This equilibrium gives the plants stocks concentrations and depends on the pace of absorption $\xi$. We assume $\xi$ to be plastic allowing plants to remain at stoichiometric balance, implying that $p_{*} / q_{*}=\alpha_{\tau}$. This assumption and eq. (A9) together fix the value of the absorption rate $\xi \sum_{i=l, r} \mathcal{E}_{i} n_{i}$. It will be convenient to write the latter as $\gamma \mathcal{A}$. With this notation:

$$
\mathcal{A}=\varphi \sum_{i=l, r} \frac{\frac{\mathcal{E}_{i} n_{i}}{m}}{1+\alpha_{\tau} \sum_{i} \frac{\mathcal{E}_{i} n_{i}}{m}}
$$

This expression proposes a natural decomposition $\mathcal{A}=\mathcal{A}_{l}+\mathcal{A}_{r}$, representing the sum of absorption rate of labile and recalcitrant N, respectively. Stoichiometric balance also implies that $\kappa\left(p_{*}, q_{*}\right)=p_{*} / \gamma$. We define $P=p_{*} / \gamma$, which corresponds to the ratio of $\mathrm{N}$-stocks over the N-content of effective biomass, $\gamma B$. From eqs. (A9-A10) it holds that

$$
P^{2}+P=\mathcal{A}
$$

Eq. (A2) defining plant growth can finally be rewritten:

$$
\frac{d B_{\tau}}{d t}=\mu(P-h) B_{\tau}
$$


Productivity (rate of biomass creation) equals $\mu P$, therefore $P$ is the effective nutrient stock which drives plant productivity in our model. At equilibrium $P=h$. If $h$ is smaller than one, meaning that turnover is relatively slow compared to biomass growth than we do have that $P \approx \mathcal{A}$. For simplicity, this what we assumed in all analytical expression of the main text.

\section{Nutrient absorption and recycling}

In the soil, we model two variables: the amounts of labile and recalcitrant nutrients ( $n_{l}$ and $n_{r}$, respectively). The dynamics of labile nutrients is controlled by three fluxes: absorption by plants, input by litter fall, and exogenous nutrient flows.

Absorption by plants is modeled as described in the previous section on plant physiological dynamics removing $\mathrm{N}$ from the soil at a rate $\mu \mathcal{A}_{l} \times \gamma B$. Litter input depends (i) on the amount of litter entering the soil and (ii) on the proportion of litter $\mathrm{N}$ that is in a labile form. The amount of litter entering the soil is proportional to turnover rate and the $\mathrm{N}$ parts of biomass, $\gamma B$, plus that of stocks, $n=\operatorname{P\gamma } B$. The proportion in labile form is a negative function of plant tannins, because tannins will complex part of nitrogen and turn it into its recalcitrant form. If one unit of tannins complexes $\Omega$ units of $N$, then we get a recycling term $\mu \mathcal{R}_{l} \times \gamma B$, with

$$
\mathcal{R}_{l}=\bar{\ell} h\left(P+1-\Omega_{\tau}\right)
$$

where $\bar{\ell}=1-\ell$ models $\mathrm{N}$ losses during decomposition (e.g. volatilization) and $\Omega_{\tau}=$ $\Omega \tau / \gamma_{\tau}$ so that $\Omega_{\tau} \gamma B$ is the amount of $N$ biomass being complexed by tannins. The dynamics of labile soil nitrogen are completed by an exogenous flow, representing natural input and output of nitrogen, in particular through leaching. We write this term as

$$
L\left(n_{w}-n_{l}\right)
$$

Where $n_{w}$ is the bare soil nutrient content (Droop, 1968; Lemesle and Mailleret, 2008) and $L$ is the leaching rate of labile N. We will take this rate to set a reference ecological time 
scale, so that henceforth $L=1$. As a result, the global equation modeling the dynamics of labile $\mathrm{N}$ in the soil is:

$$
\frac{d n_{l}}{d t}=\mu(\underbrace{\mathcal{R}_{l}}_{\text {recycling }}-\underbrace{\mathcal{A}_{l}}_{\text {absorption }}) \gamma B+\underbrace{n_{w}-n_{l}}_{\text {in and out flows }}
$$

What $\mathrm{N}$ is complexed by tannins flows into the recalcitrant compartment $n_{r}$. This is the only inflow in $n_{r}$. Similarly as the one of labile $\mathrm{N}$, the dynamics of $n_{r}$ thus read

$$
\frac{d n_{r}}{d t}=+\mu(\underbrace{\mathcal{\mathcal { R } _ { r }}}_{\text {recycling }}-\underbrace{\mathcal{\mathcal { A } _ { r }}}_{\text {absorption }}) \gamma B-\underbrace{\mathcal{L} n_{r}}_{\text {leaching }}
$$

$\mathcal{R}_{r}=\Omega_{\tau}$. Note that symbiotic capacity $e$ only enters in the absorption terms, via the benefit to ratio $\mathcal{E}_{i} / m$. The mean-field model is determined by the system of ODEs (5-6-7) complemented by eqs. 3 and 4 .

\section{Appendix B: details of the cellular automaton}

Space was represented as a landscape of S patches, which can be either empty or occupied by an individual plant. Individuals grow according to the above model, absorbing and recycling $\mathrm{N}$ locally. But, in contrast to the mean-field model, $\mathrm{N}$ diffuses continuously across patches. For a general topology of connected patches the diffusion term affecting nutrients in patch $k$, either labile $n_{l}^{(k)}$ or recalcitrant $n_{r}^{(k)}$, reads,

$$
\mathcal{D}_{i}\left(\sum_{p \in N e i(k)} n_{i}^{(p)}-\# N e i(k) n_{i}^{(k)}\right)
$$

where $\operatorname{Nei}(k)$ denotes the set of neighbors of patch $k$ and $\mathcal{D}_{i}, i=l, r$, are diffusion rates. The first term (the sum) represents in-flow from neighboring patches of patch $k$ while the second represents out-flows from patch $k$ towards its neighbors. In simulations we considered the simplest possible landscape: a one-dimensional periodic lattice, so that $\operatorname{Nei}(k)=\{k-1, k+1\} \bmod (S)$. We will typically assume $\mathcal{D}_{l}$ to be much larger than $\mathcal{D}_{r}$, making recalcitrant $\mathrm{N}$ less mobile. We then modeled population dynamics. This was 


\begin{tabular}{|c|c|c|}
\hline Parameter & interpretation & value \\
\hline$\alpha$ & $\mathrm{C}: \mathrm{N}$ ratio of effective biomass & 40. \\
\hline$\mu$ & metabolic rate & 5 \\
\hline$h$ & relative turnover rate & 0.6 \\
\hline$H$ & relative herbivory rate & 0 or 0.01 \\
\hline $\mathcal{E}_{l}^{-}$ & min labile absorption efficiency & 1 \\
\hline $\mathcal{E}_{l}^{+}$ & max labile absorption efficiency & 2 or 2.1 \\
\hline $\mathcal{E}_{r}^{+}$ & max recalcitrant absorption efficiency & 1.1 \\
\hline$m_{0}$ & min maintenance cost & 1 \\
\hline$\varphi_{0}$ & max photosynthesis rate & 100 \\
\hline$\ell$ & $\mathrm{N}$ volatilization & 0.2 \\
\hline$n_{w}$ & bare soil $\mathrm{N}$ content & 1 \\
\hline $\mathcal{N}$ & effective bare soil $\mathrm{N}$ content & 0.6 \\
\hline $\mathcal{L}$ & recalcitrant leaching rate & 0.1 \\
\hline$\Omega$ & Tannins complexation power & 1 \\
\hline $\mathcal{D}_{l}$ & labile diffusion rate & 10 \\
\hline $\mathcal{D}_{r}$ & recalcitrant diffusion rate & 0.1 \\
\hline$r_{\text {birth }}^{+}$ & max birth rate & tuned to \\
\hline$r_{\text {death }}^{+}$ & max death rate & avoid \\
\hline$\lambda$ & demographic effect of biomass & neutrality \\
\hline K & seed dispersal kernel & 0.6 \\
\hline$\delta$ & fraction of seeds uniformly dispersed & 0.2 \\
\hline$z$ & phenomenological local mass parameter & 0 or 0.03 \\
\hline
\end{tabular}

Table A1: Model parameters (in order of appearance). Rates are expressed relatively to labile leaching rate. Similarly, the biomass density of plants that saturate photosynthesis sets the mass scale. Recall that the other stoichiometric parameters $\gamma, \alpha_{\tau}$ and $\gamma_{\tau}$ are explicit functions of $\alpha$ and $\tau$. 
implemented by allowing individuals to randomly die and/or reproduce in an empty patch. We assume that larger individuals are less likely to die than smaller ones and, similarly, that production of seedlings increases with biomass. Concretely, we set

$$
r_{\text {death }}\left(B_{\tau}\right)=r_{\text {death }}^{+} e^{-\lambda B_{\tau}} ; r_{\text {birth }}\left(B_{\tau}\right)=r_{\text {birth }}^{+}\left(1-e^{-\lambda B_{\tau}}\right)
$$

Newly produced plants colonize empty patches. Due to limited dispersal, the probability of establishment of a seedling is higher in patches located near the parent tree and lower for distant patches. Concretely, we write the recruitment rate in an empty patch $k$ as

$$
r_{\text {recruitment }}(k)=\sum_{d>0} F(d) \sum_{p \in N e i^{(d)}(k)} \frac{r_{\text {birth }}\left(x_{\tau}(p)\right)}{\# N e i^{(d)}(p)}
$$

where $F(d)$ is the fraction of a plant reproductive effort that will result in colonizing patches located at distance $d$ from this parent plant $\left(\mathrm{Nei}^{(d)}(k)\right.$ is the set of patches at distance $d$ from patch $k$ ). In simulations we will superpose a geometrically decreasing kernel up to $d=3$ to a uniform contribution of the remaining effort to all other patches, representing the fraction of seeds transported by the wind or by animals to arbitrarily distant locations. Concretely, for some $\delta, K<1, F(1)=(1-\delta) K, F(2)=(1-\delta) K(1-K)$, $F(3)=(1-\delta)(1-K)^{2}$ to which we add a mean-field contribution $\frac{\delta}{S} \sum_{p} r_{\text {birth }}\left(x_{\tau}(p)\right)$.

When a new plant is recruited in a patch, the plant starts growing from a small initial biomass. Generally, the demographic parameters were tuned to always have a densely populated landscape. Indeed, if many patches are empty at any given time, the dynamics become neutral and we cannot expect to exhibit selective pressures (see Table A1).

\section{Appendix C: The three steps of the model analysis}

\section{First step: implicit spatial structure}

Suppose that tannins affect the soil composition surrounding one or a clustered group of mutants. We account for this by defining a parameter $z$, an implicit function of spatial 
processes controlling aggregation of mutants and the diffusion of the recalcitrant $\mathrm{N}$ that they produce. To define $z$, we start from $n_{r}^{\tau}$, the amount of recalcitrant $\mathrm{N}$ formed in the vicinity of a mutant individual, or group, with biomass $B_{\text {mut }}$. This $\mathrm{N}$ content should be proportional to $\tau B_{\text {mut }}$, the $C$-amount of tannins produced by the mutants. This will indeed induce an inflow into the local pool $n_{r}^{\tau}$ proportional to $\tau B_{\text {mut }} \times \mu h$, where $\mu h$ is the absolute biomass turnover rate. At the same time, this pool will suffer a loss at a rate proportional to leaching and diffusion. Keeping track of relative turnover $h$, and tannins $\tau$, we define $z$ so that $n_{r}^{\tau}=z h \tau$. This phenomenological mass parameter will enable us to tune the direct effect that mutants, by changing their N-environment, have on their absorption effort $\mathcal{A}$. Concretely we evaluate eq. 8 at $\tau=0$, with a change in absorption

$$
\left.\partial_{\tau}\right|_{\tau=0} \mathcal{A}=\left.\partial_{\tau}\left\{\varphi \frac{\frac{\mathcal{E}_{l} n_{l}}{m}+h \tau \frac{\mathcal{E}_{r} z}{m}}{1+\alpha_{\tau}\left(\frac{\mathcal{E}_{l} n_{l}}{m}+h \tau \frac{\mathcal{E}_{r} z}{m}\right)}\right\}\right|_{\tau=0}
$$

evaluated at the equilibrium values of the resident. Note that setting $z=0$ corresponds to the mean-field model in which there is no spatial structure. This analysis answers the question: - Given that mutants modify their local environment (as measured by z), under which conditions will this allow them to invade a resident population, and thus lead to the selection of the tannin phenotype?

\section{Second step: explicit spatial structure}

We used the cellular automaton to test the results of the implicit approach described above. We simulated invasion attempts of tannin-producing plants (with $\tau=0.2$ ) in a landscape populated by tannin-less residents. The landscape was a periodic lattice comprised of $S=250$ cells. Invasions started from 5 spatially aggregated individuals. We monitored the ultimate fraction of mutants after 400 time steps. A snapshot of a run of the automaton is presented in Fig 2, where we can see the impact of tannin producing individuals on soil variables surrounding their respective patches: We then focused on social effects allowed by spatial structure. 
1) To relate tannin evolution to the evolution of altruism in a spatial context (van Baalen and Rand, 1998), we ran invasion attempts along a gradient of values of recalcitrant diffusion rate $\mathcal{D}_{r}$, and estimated the probability of a successful invasion as a function of $\mathcal{D}_{r}$. For low diffusion, mutant individuals are effectively "selfish": they do no share their recalcitrant N-pool with neighboring plants. By contrast they share it with all plants if diffusion is large. In this latter case we expect a "tragedy of the commons", where the resident plants, seen as "cheaters", benefit without costs from the complexed $\mathrm{N}$ pool so that tannins are not selected. For intermediate diffusion, a tannin producing individual shares his complexed $\mathrm{N}$ pool with a few other individuals that are likely to be of his kin. If invasion is more likely then at $\mathcal{D}_{r}=0$, this would demonstrate the contribution of group selection, a mechanism that can allow the selection of pure altruism (van Baalen and Rand, 1998; Wilson, 1980).

2) The death of a tannin producing individual leaves a N-rich patch available for recruitment from neighbors that are also likely to be of his kin. In this case individuals inherit the environment transformed by their ancestors, a property termed ecological inheritance (Odling-Smee et al., 2003). To test if ecological inheritance played a role in our system, we devised an arbitrary way to turn it off: following the death of a tannin producing plant, we set to zero the recalcitrant $\mathrm{N}$ pool that it had created (i.e. $\mathrm{N}$ pools vanish before the establishment of new seedlings). This requires diffusion of recalcitrant $\mathrm{N}$ to be negligible, otherwise it becomes impossible to determine the part of $n_{r}$ that is associated to the individual plant that died. Thus, setting $\mathcal{D}_{r}=0$ we monitored the ultimate relative abundance of mutants, with and without ecological inheritance.

\section{Third step: coevolution of tannins and symbiotic capacity}

For any resident phenotype $(e, \tau)$, we computed the ecological equilibrium of the meanfield model. We then determined the fitness gradient at $(e, \tau)$ by evaluating the initial growth rate of slightly different mutant phenotypes $(e+\delta e, \tau+\delta \tau)$. The fitness gradient 
has now two components, one in the direction of symbiotic capacity and one in the direction of tannins:

$$
\left.\nabla W\right|_{e, \tau}=\left(\left.\partial_{e} W\right|_{e, \tau} ;\left.\partial_{\tau} W\right|_{e, \tau}\right)
$$

Coevolutionary trajectories follow the direction of the fitness gradient until it vanishes and a steady state is reached (Leimar, 2009). For the evolution of single traits, such steady states are called CCS, Continuously Stable Strategies (Eshel, 1983; Geritz et al., 1998); in a coevolutionary context we will refer to a co-CSS.

Similarly as in eq. 8 from the invasibility analysis, the first component of the fitness gradient is proportional to $\partial_{e} \mathcal{A}$, the change of $\mathrm{N}$-absorption as $e$ is varied. In our model, this change is driven by nondimensional ratios $\frac{\mathcal{E}_{i} n_{i}}{m}$ representing the effective fertility of the soil as perceived by the plants. They read

$$
\begin{aligned}
\frac{\mathcal{E}_{l} n_{l}}{m} & =\left((1-e) \frac{\mathcal{E}_{l}^{-}}{\mathcal{E}_{l}^{+}}+e\right)(1-e) \frac{\mathcal{E}_{l}^{+} n_{l}}{m_{0}} \\
\frac{\mathcal{E}_{r} n_{r}}{m} & =e(1-e) \frac{\mathcal{E}_{r}^{+} n_{r}}{m_{0}}
\end{aligned}
$$

They are non monotonous functions of $e$, meaning that there is a trade-off between increasing efficiency and reducing C-cost.

The second component of the fitness gradient is proportional to $\partial_{\tau} \mathcal{A}-\partial_{\tau} h$, where $\partial_{\tau} h$ is non zero only in the presence of herbivores. The absorption term depends, in particular, on the way mutants change their local N-environment. To model the outcome of this change, we used eq. C1 which implicitly accounted for spatial structure via the phenomenological mass parameter $z$. We slightly generalized the approach to allow for a tannin producing resident. We wrote the recalcitrant $\mathrm{N}$-content in the vicinity of an aggregated group of mutants as

$$
n_{r}^{\tau+\delta \tau}=n_{r}^{*}+z h \delta \tau
$$

where $n_{r}^{*}$ is recalcitrant content produced by the tannins of the resident plants. From there we applied the same reasoning as in eq. C1 to compute $\partial_{\tau} \mathcal{A}$. The co-CSS will corre- 
spond to an intersection between the isocline of symbiotic capacity $\left\{(e, \tau)\left|\partial_{e} W\right|_{e, \tau}=0\right\}$ and the one of tannin content $\left\{(e, \tau)\left|\partial_{\tau} W\right|_{e, \tau}=0\right\}$. We first chose the labile absorption efficiency $\mathcal{E}_{l}$ so that symbiotic capacities could be favored in the absence of tannins by enhancing labile absorption. We asked whether this could then initiate the evolution of tannins, and if so, if this would further promote symbiotic capacity. By contrast, we considered the case where herbivory could favor tannin production but where symbiotic capacity could not evolve without tannins. We asked whether the evolution of tannins as an anti-herbivore defense could then promote the evolution of symbiotic capacity and then further promote tannin production.

\section{Appendix D: derivation of the fitness gradient in the implicit ap- proach. A mathematical explanation of the results of Fig. 3.}

For completeness we provide a computation of the fitness gradient. This will show more explicitly the role of all model parameters on the fitness of the Tannin phenotype and explain the mathematical origins of the plots shown in Fig. 3. We start from

$$
\partial_{\tau} W=\partial_{\tau} \mathcal{A}-\partial_{\tau} h
$$

We have that $\partial_{\tau} h=-H$ where $H$ is the relative rate of herbivory. Less trivial is the term $\partial_{\tau} \mathcal{A}$. For conciseness we first define $\mathcal{N}_{l}=\frac{\mathcal{E}_{l} n_{l}}{m}$ and $\mathcal{N}_{r}=\frac{\mathcal{E}_{r} z}{m}$. We have that

$$
\partial_{\tau} \mathcal{A}=\partial_{\tau}\left\{\varphi \frac{\mathcal{N}_{l}+h \tau \mathcal{N}_{r}}{1+\alpha_{\tau}\left(\mathcal{N}_{l}+h \tau \mathcal{N}_{r}\right)}\right\}
$$

evaluated at $\tau=0$ and at the ecological equilibrium state determined by the resident plant population, namely $\varphi \frac{\mathcal{N}_{l}}{1+\alpha \mathcal{N}_{l}}=h$. We first note that, at first order in $\tau, \alpha_{\tau}=\alpha+\tau$. Thus keeping track of first order terms we get

$$
\mathcal{A}=\frac{\varphi \mathcal{N}_{l}}{\underbrace{1+\alpha \mathcal{N}_{l}}_{=h}} \frac{1+h \tau \frac{\mathcal{N}_{r}}{\mathcal{N}_{l}}}{1+\tau \underbrace{\frac{\mathcal{N}_{l}}{1+\alpha \mathcal{N}_{l}}}_{=h / \varphi}(1+\alpha h \underbrace{\frac{\mathcal{N}_{r}}{\mathcal{N}_{l}}}_{=z \mathcal{E}_{r} / \mathcal{E}_{l}})}
$$


so that

$$
\mathcal{A}=h\left\{1+\tau h\left(\left(1-\frac{\alpha h}{\varphi}\right) \frac{\mathcal{N}_{r}}{\mathcal{N}_{l}}-\frac{1}{\varphi}\right)\right\}
$$

thus

$$
\partial_{\tau} \mathcal{A}=h\left\{\left(1-\frac{\alpha h}{\varphi}\right) \frac{\mathcal{E}_{r} z}{\mathcal{E}_{l}}-\frac{1}{\varphi}\right\}
$$

here we can understand the hump-shaped relationship of fitness with turnover $h$ seen in Fig. 3. Indeed, we see that high $h$ will eventually reduce the increase of absorption, due to the term $-\frac{\alpha h}{\varphi}$. However, low $h$ is not optimal either because this will imply a build up in plant biomass $B$, leading to a reduction of photosynthesis rate $\varphi(B)=\frac{\varphi_{0}}{1+B}$. We deduce that, in terms of tannin fitness, there exist an optimal, intermediate value of turnover.

Soil fertility $\mathcal{N}=\frac{\mathcal{E}_{l} n_{w}}{m}$ enters via the labile absorption efficiency $\mathcal{E}_{l}$ and indirectly in the photosynthesis rate $\varphi$. Indeed, all else being equal, lower soil fertility implies lower plant biomass thus relaxes competition for light. We thus see that reducing fertility is always beneficial for tannin production.

In the presence of herbivores, the factor $h$ in the above expression for $\partial_{\tau} \mathcal{A}$, which is absent in the term $\partial_{\tau} h=H$ tells us that, at low turnover rates, it is the protection against herbivores that determines tannins' fitenss and thus their evolution. 